\title{
Entdeckung einer
}

\section{metallurgischen}

\section{Industrie}

am Fusse des

Hochhimalajas.

Prospektionsbericht und Schlussreport der Probenuntersuchungen einer Expedition während der Drapham-Dzong-Grabung in Bhutan/Provinz Bumtang der Universität Basel (2008 - 2010)

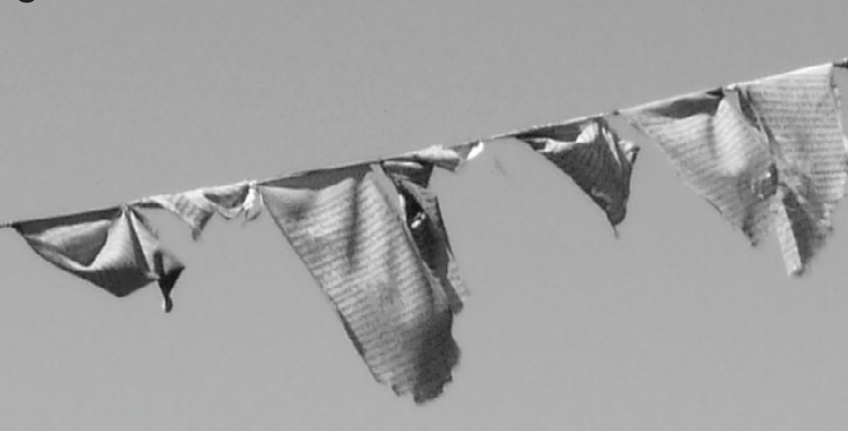


Entdeckung einer metallurgischen Industrie am Fusse des Hochhimalajas. 


\section{Impressum}

○ 2013 by LIBRUM Publishers \& Editors LLC, Hochwald (Schweiz).

Alle Rechte vorbehalten. All rights reserved.

Jede Art der Vervielfältigung ohne Genehmigung des Verlages ist unzulässig.

Übersetzung Deutsch - Englisch: Anne Waite

Übersetzung Französisch - Deutsch: Dominique Oppler

Layout und Satz: Fanny und Lukas Oppler

ISBN: 978-3-9524038-0-8

Umschlagabbildung:

Blick vom Drapham-Dzong zu den Bergen des Hochhimalajas,

Foto D. Oppler

www.librum-publishers.com

\section{(c) $(1) \Theta$}

Entdeckung einer metallurgischen Industrie am Fusse des Hochhimalajas by Dominique Oppler, Raphaëlle Soulignac, Vincent Serneels is licensed under a Creative Commons Attribution-NonCommercial-NoDerivatives 4.0 International License. 
Entdeckung einer metallurgischen

Industrie

am Fusse des

Hochhimalajas. 


\section{Inhaltsverzeichnis}

Vorwort

Summary . . . .

Prospektionsbericht ….............................................. 13

Analyse der Schlackenproben ………………............ 23

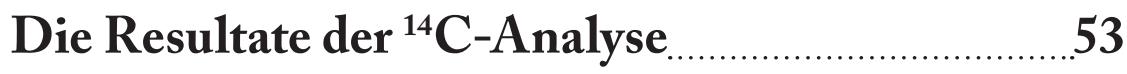

Schlussfolgerung

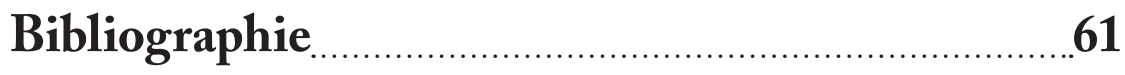

Anhang $\ldots 5$ 


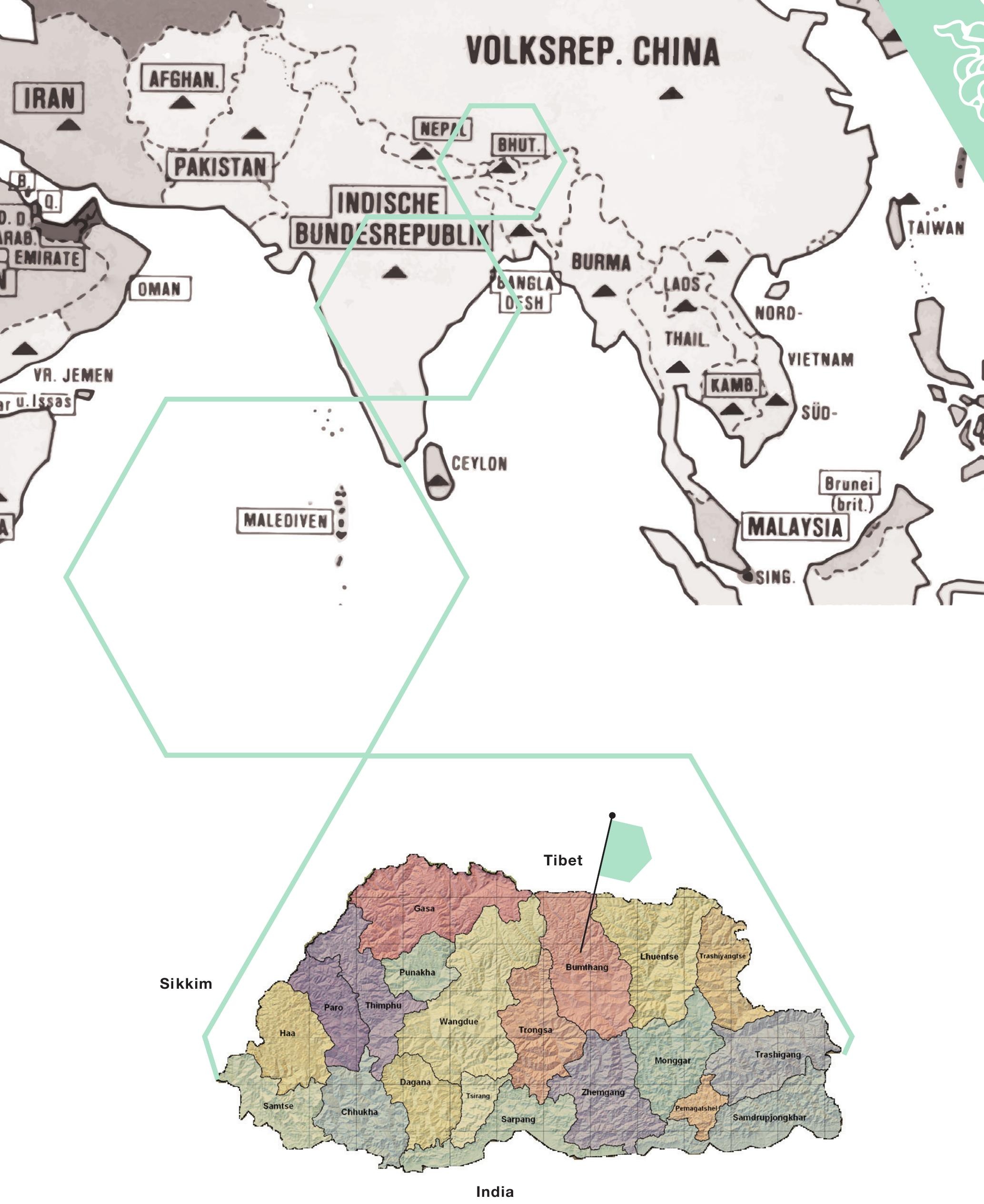




\section{Vorwort}

\section{Warum Archäologie in Bhutan?}

Bhutan ist ein relativ junger Staat. Bis ins späte 17. Jh. war das Land nicht geeint. Die Regionen und Täler wurden von regionalen Königen und Fürsten regiert. Die Oberherrschaft teilten sich bis Ende des 19. Jh. ein religiöser und ein weltlicher Monarch. 1907 setzte sich die Familie der Wangchucks durch und errichtete eine Erbmonarchie. Derzeit regiert der V. König, Jigme Khesar Namgyel Wangchuck, ca. 30 jähriger Sohn des IV. Königs, der 2006 vorzeitig die Regierungsgeschäfte seinem Sohn übertragen hat. Zuvor aber wurde die Regierungsverantwortung umstrukturiert und eine konstitutionelle Monarchie nach britischem Vorbild eingerichtet.

Dieser Demokratisierungsprozess ist noch im Gang. Zwar ist der erste, demokratisch gewählte Premier im Amt, und auch zahlreiche Minister auf ebenso demokratischem Weg gewählt worden, doch wenn man die Situation etwas näher betrachtet, so fällt auf, dass Verwandtschaftsgrade die Wahlresultate erheblich beeinflusst haben dürften. Der heutige Premier-Minister ist beispielsweise der Schwager des letzten Premiers, welcher noch unter der Regentschaft des IV. Königs amtierte, usw.

Aber die Bhutanesen kümmert es wenig, denn der Bevölkerung geht es relativ gut. Es herrscht zwar materielle Armut im ganzen Land, doch alle haben zu Essen. Grund dafür sind die noch intakten sozialen Strukturen mit Gross-Familien, 
Sippen, Clans und Talgemeinschaften, die ein breit abgestütztes Netz bilden, das sich selbst versorgt und in allen Lebenslagen jedes Mitglied auf seine Weise stützt. Die Bhutanesen nehmen relativ passiv am politischen Geschehen teil. Das Land ist verkehrstechnisch schlecht erschlossen. Viele Verkehrsachsen sind zwar geteert, doch sie führen entlang der Höhenkurven von Berg- und Hügelzügen. Eine Fahrt von einem Ort zum anderen, auch wenn diese luftlinienmässig nahe bei einander liegen, dauert oft Stunden oder Tage. Autobahnen kennt man nicht. Eisenbahn oder einen nationalen, regionalen Flugverkehr ebensowenig. Paro ist ${ }^{1}$ der einzige Flughafen, mit welchem Bhutan auf dem Luftweg mit der übrigen Welt, insbesondere mit Indien, verbunden ist. So bleiben also das Fahrzeug, wovon die wenigsten eines besitzen, das Maultier, das Yack oder die eigenen Beine und Schultern, welche zum „Reisen“ oder Schleppen von Lasten zur Verfügung stehen. Und eines können die Bhutanesen: Schleppen!

Hinzu kommt, dass Medien wie TV, Radio, Internet, aber auch Zeitungen, nur in den Hauptorten eine gute Distribution haben, in den Landregionen jedoch kaum Einsatz finden - mangels Elektrizität, mangels regelmässig funktionierendem Postsystem, etc.

Diese Situation führt dazu, dass die Täler meist auf sich selbst gestellt und mit ihren eigenen, unmittelbaren Sorgen beschäftigt sind. Mit nationalen, geschweige denn internationalen Impulsen, kommen sie kaum in Berührung.

Es liegt auf der Hand, dass ein demokratischer Staat es auf diese Weise schwer hat, seine Bevölkerung für die nationalen Interessen zu begeistern und zu mobilisieren.

Ein weiterer Aspekt ist ethnologischer Art - „the present is the key to the past”. Die Bevölkerung Bhutans ist ethnisch sehr durchmischt, denn die Geschichte des bhutanesischen Staates ist auch eine Geschichte Tibets, Nepals und Nordindiens. Das Gebiet wurde mehrfach besetzt und zurückerobert. Durch Bhutan führen zahlreiche Handelswege, von Norden nach Süden und von Osten nach Westen. Das Volk ist teils sesshaft, teils nomadisch. Ein nationales Bewusstsein entwickelt sich erst, und an diesem Punkt setzt der Staat nun an: man ist auf der Suche nach der „eigenen“ Geschichte, einer „eigenen“ Identität.

Das tantrische, lamaistische Bhutan kennt (noch) sehr wenig eigene Quellen zur Geschichte des eigenen Landes. Viele Überlieferungen sind religiös, mythisch, philosophisch begründet und es kursieren zahlreiche Legenden und oral histories. Die einzig bekannten historisch belegten Quellen stammen aus Tibet, Nepal oder Indien. Doch gibt man sich mit Recht damit nicht zufrieden, denn Geschichte war und ist Spielball politischer Rechtfertigung. So setzt man also auf eigene Geschichtsschreibung und mangels verlässlicher Quellen, hat sich Bhutan der Archäologie verschrieben. 
Der Impuls zu archäologischen Grabungstätigkeit in Bhutan geht also vom Staat Bhutan selbst aus. Weil die politischen, wirtschaftlichen und vor allem karitativen Beziehungen zur Schweiz sehr eng sind, hat man dort angeklopft.

Das Ziel Bhutans ist eine eigene Landesarchäologie aufzubauen. Hierzu wurde ein Pilotprojekt entwickelt, das dem Land als „Lehrstück“ dienen soll: Die Grabung auf dem Drapham-Dzong.

Die wissenschaftliche Leitung dieses Projektes obliegt der Universität Basel, em. Prof. Dr. Werner Meyer. Die Finanzierung wurde durch den Staat Bhutan, dem Schweizerischen Nationalfonds, der Schweizerisch-Liechtensteinischen Stiftung für Archäologie (SLSA), der Akademischen-Gesellschaft Basel, der Gesellschaft Rietberg-Museum Zürich und der Helvetas sichergestellt.

Parallel hierzu wurde eine Frau aus Bhutan, Kuenga Wangmo, nach Cambridge zum Archäologie-Studium entsannt. Sie ist bestimmt, später die Leitung der Landesarchäologie Bhutans zu übernehmen.

\section{Der Drapham-Dzong in Bumthang}

2006/07 wurde in der Provinz Bumthang der von Bhutan vorgeschlagene Ort für die erste archäologische Grabung im Land prospektiert: Die Burgstelle des Drapham-Dzong.

Ein Dzong ist eine Klosterburg, ein feudaler Sitz und gleichzeitig eine Festung. Dzongs bilden religiöse und herrschaftliche Zentren, sind Verwaltungssitz eines Distrikts. Es war früher durchaus üblich, dass in einem Dzong sowohl Mönche wie auch der Monarch gleichzeitig lebten, und noch heute bilden Religion und Tagesgeschäft bei allen Bevölkerungsschichten eine untrennbare Einheit.

In Bhutan sind noch ca. 20 Dzongs in Betrieb, die meisten stammen aus dem 17.Jh. Eine vergleichbare Dzong-Tradition und Dzong-Architektur kennt man ebenso in Tibet, Ladakh und Sikkim.

Aufgrund erster Ergebnisse der Radiocarbon-Analyse, und zahlreichen mündlichen Überlieferungen, weist der Drapham-Dzong in die Zeit des 15. und 16. Jh., also einer sehr frühen Phase der Dzong-Bautätigkeiten, vielleicht handelt es sich sogar um den ersten Dzong auf bhutanesischem Gebiet überhaupt. Die Ausdehnung des Drapham-Dzong ist zudem die grösste bisher bekannte Festung Bhutans. Die Burg ist in einer geologisch höchst spektakulären Gegend, auf einem ca. $100 \mathrm{~m}$ hohen, steilabfallenden, kegelförmigen Hügel errichtet, welcher nord-östlich von einem kleineren Bach, nord-westlich von einem reissenden Fluss umspült wird. 
Auf dem höchsten Punkt dieses Hügels stand die Hauptburg. Sie ist umgeben von einer grosszügigen Vorburg, womöglich auch von einem Zwinger, zahlreichen Wachttürmen, einem befestigten Wasserweg, welcher zum Bachlauf am Fusse des Hügels führt, sowie eines eigenen Siedlungsplatzes (ev. für Handwerksbetriebe), der Teil der Vorburg bildete. Alles in allem ein Areal das sich über ca. 15 ha Luftlinie erstreckt.

Reichtum ist die Grundlage, damit eine monumentale Anlage wie jene des Drapham-Dzongs errichtet und unterhalten werden konnte. Doch woher stammte dieser Reichtum, welches waren die strategischen Gründe, dass ausgerechnet in einer der ärmsten Gegenden Bhutans, der Provinz Bumthang, diese Burg errichtet wurde?

Die nachfolgenden Ausführungen und Untersuchungsresultate versuchen zur Beantwortung dieser Fragestellung beizutragen.

\section{Dank}

Mein Dank geht zu aller erst an em. Prof. Dr. Werner Meyer, dem ich zu verdanken habe, dass ich der Grabungskampagne 2010 beiwohnen durfte, der mir das Vertrauen schenkte, die kleine Expedition zum Schlackenplatz anzuführen und mich auch mit den metallurgischen Analysen und dem Verfassen des Schlussberichtes betraute.

Auch danke ich der Projektleiterin, Silvia Scheuerer und dem Grabungsteam für die tolle Zusammenarbeit während und nach der Grabung.

Dr. Ludwig Eschenlohr, welcher mich während zwei Grabungen in der Schweiz in die Geheimnisse der Metallurgie einführte und als erster einen fachkundigen Blick auf die Probensammlung warf, sei herzlich gedankt.

Dr. Kuenga Wangmo verdanke ich die Verifizierung ethnologisch-, historisch- und religionsrelevanter Informationen.

Die vertieften metallurgischen Untersuchungen verdanke ich Prof. Dr. Vincent Serneels und Raphaëlle Souligniac, welche mir das Labor der Geoscience an der Université de Fribourg zur Verfügung stellten und mich bei der Probenaufbereitung, der Analyse und Interpretation fachkundig begleiteten.

Ebenso gebührt grossen Dank an Dr. Irka Hajdas und dem Team des ${ }^{14} \mathrm{C}$-Labors an der ETH Zürich, für die Unterstützung beim Aufbereiten der ${ }^{14} \mathrm{C}$-Proben und die Interpretation der Daten.

Dominique Oppler 


\section{Summary}

During the archaeological campaign of the University of Basle at the Drapham-Dzong in Bhutan from 2008 to 2010, iron slag came to light inside the 15th or 16th century monastery-castle. Moreover, in the bamboo jungle of the nearby hamlet Nag Lhakhang, a metal producing locality, a slagheap and a platform, which could possibly be the site of an ancient charcoal burner, were found. This roused the issue of whether the iron smelting stands in context with the wealth of the monastery-castle.

The sites are around $14 \mathrm{~km}$ from Jakar, in the province Bumtang, above Nag Lhakhang (2824 $\mathrm{m} \mathrm{ASL}$ ), in a little side valley with gentle slopes, and two little mountain streams. (Fig. 1 - 2)

This valley has dry meadows used as pasture. The humous soil is thin and just covers the stony or rocky substrate. The small range of hills is thickly forested with hardwood and coniferous trees and only reached with considerable difficulty. The "charcoal burner site", the place of metal production and the slagheap lie on an East-West axis over $80 \mathrm{~m}$ in length. (Fig. 3) 


\section{The place of metallurgical production - a bloomery furnace}

In a cleared surface of $6 \times 6 \mathrm{~m}$, a natural stone accumulation covers an area of 5 $x 5 \mathrm{~m}$ and gives the impression of a collapsed structure. There were no detectable constructions.

On the northeast side of the stone accumulation, the stones overlay the topsoil by over $50 \mathrm{~cm}$ in an area of 2 by $5 \mathrm{~m}$. In the southerly direction, and therefore in the direction of the slagheap, the number of overlaid stones decrease and are situated in such a way that an outflow could be presumed.

Through diverse fissures in the middle of the upper third of the stone accumulation, we measured a depth of at least $60 \mathrm{~cm}$, which suggests a pit into which the stones have collapsed.

Most of the stones are natural, probably granitic and of different sizes. Traces of machining cannot be excluded. The stones in the area of the "outflow" as well as those in the middle of the main accumulation show red discolorations. Abundant accumulations of slags were found on both sides and in the extension of the "outflow".

Further analysis will reveal that what we found at this place is a bloomery furnace. (Fig. 4 - 5)

\section{The slagheap}

The slagheap is situated $16 \mathrm{~m}$ below the production place. The slag emerges at the edge of an elevated platform and forms a heap of $30 \mathrm{~m}$ by $6 \mathrm{~m}$ (Fig. 6). There is a strikingly large amount of huge, heavy, dense and highly oxidised grey-denseslag (GDS), with no flow patterns and an avarage size of $20-30 \mathrm{~cm}$ (Fig. 7). Most of this slag shows pronounced traces of wood or charcoal. Due to high freight costs, we had to limit the size of the samples.

Among the little slag fragments we collected at the heap, we found slag type GDS with marked flow traces as well as GPS (grey-porous-slag), glazed, very porous and whitish to white-grey; these were similar to the samples we found at the metallurgical production place. Also we found fragments of fired clay, which was partially slagged. We did not find any calotte.

Between the slags we also observed natural stones as we have already described 
at the metallurgical production place, slagged and with red discolorations.

We may assume, that the slag volume of the heap does not correspond to the effective quantity of production waste because the slag has a marked importance to the population. There is a belief that the metallurgical industry of this site goes back to the legends of the holy Lama Terton Pema Lingpa. The slags are therefore traded as relics, which bring happiness to the court and homes. As we could observe in the hamlet Nag Lhakhang, the slag had been removed in considerable quantities. We have no knowledge of the period over which this trade has taken place.

We searched other areas and explored the little creeks but did not found other places of interest. For reasons of time and lack of authorisation, the assumed site of the charcoal burner could not be examined.

\section{Analyses of the samples}

We took 58 samples $(7,5 \mathrm{~kg})$ in total, which include 11 from the bloomery furnance, 43 from the slagheap and 2 from the Drapham-Dzong excavation.

All samples were washed, macroscopically described, classified and sketched, measured, and weighed. The density was also determined.

24 samples were subjected to an in-depth analysis, which include 5 samples from the bloomery furnace $(\mathrm{O}), 17$ samples from the slag heap $(\mathrm{H})$ and 2 samples from the Drapham-Dzong excavation. The samples were sketched and halved with a diamond saw and the cut surfaces were traced and also sketched. The sawn halves of 19 pieces were ground and allocated to the XRD- and XRF-analysis.

While sawing the slag sample $\mathrm{H} 107$, well preserved charcoal pieces came to light. There were also very thin charcoal pieces adhering to the slag sample $\mathrm{Na}$, which came from the excavation of Drapham-Dzong. These charcoals were examined in the 14C-laboratory of Beam Physics at the ETH Zürich. All other analyses were were carried out at the geoscience-laboratories of the University of Fribourg (Switzerland).

Based on the macroscopic description we could determine 3 material groups:

- Grey-dense-slags (GDS), fayalitic, partially with high iron content.

- Grey-porous-slags (GPS), glazed, very porous, whitish to white-grey.

- Loamy and sandy material, more or less melted, partially with stone or rock inclusions. These may be wall fragments or an aeration nozzle, which have been exposed to high temperatures, achieving their melting point.

From each group we processed one sample into thinly-ground samples for the microscopy analysis. 
We should remember that we analysed stray finds of a brief survey only, without vertical or horizontal stratigraphical context. For this reason, the sampling cannot be representative but gives us some indications of the first metallurgical industry to be discovered at the foot of the Himalayas. We hope to go back in the near future and to get the authorisation for an archaeological excavation of this site.

\section{Conclusions of the chemico-mineralo- gical analysis}

\section{GDS-Samples}

All 7 analysed GDS-samples contain a high level of fayalite Fe2SiO4), the ironsilicate which usually is present in ferruginous slags. Fayalite is associated with spinel which is rich in aluminium. The composition of this spinel seems to be lightly variable. In some samples hercynite ( $\mathrm{Al} 2 \mathrm{FeO} 4)$ is indicated, but aluminiumpotassium sulfate or leucite (KAISiO6) is also present.

In 6 of the 7 samples we could detect iron oxides. We found the mineral hematite (Fe2O3) (3times), magnetite (Fe3O4) (3times) and wüstite (FeO) (3times). The coexistence of these three minerals is often observed in metallurgical waste and is due to high variability of the conditions of reduction in furnaces, primarily during the cooling-off period. When the cooling-off is combined with oxygen, magnetite and hematite will form at the surface.

There is a small quantity of quartz (SiO2) indicated in four samples. Due to the presence of leucite in all samples, the proportion of quartz will not have a significant influence on liquefaction. We may assume that these solid grains have a secondary origin, coming either from the wall or from the ground incorporated during outflow. They have undergone no fusion.

The association of fayalite, wüstite and leucite indicates temperatures of $1250^{\circ} \mathrm{C}$. This mineralogical constitution is usual in iron smelting slags. The high amount of iron oxide indicates a low reduction.

\section{GPS-samples}

The high amount of amorphous material within the GPS-samples draws attention to a smelting process with high temperatures.

All samples indicate a high quartz content. Mullite is consistently present even 
though the measured peaks are very low. Either the measured quantity was low or the grains have crystallized poorly. Mullite is an aluminium silicate formed at high temperatures $\left(>1100^{\circ} \mathrm{C}\right)$.

In one sample, cristobalite is manifested, a polymorphic, high-temperaturemodified silicate dioxide ( $\mathrm{SiO} 2$ ).

In three samples we identified indialite. This is a rare mineral, an aluminium-/magnesium silicate, which is formed at high temperatures during the reduction process.

But the presence of this mineral has first to be confirmed before the temperatures achieved during the process can be assessed.

Iron oxides were found in 4 samples. Hematite was identified in 3 samples and magnetite in 1 sample.

\section{Loamy-sandy material}

In both loamy-sandy samples we identified a high quartz content but a low microcline content from the feldspar family. Both samples contain hematite. The peak in sample $\mathrm{H} 102$ (red) was considerably higher than that in sample $\mathrm{H} 100$ (whitish). The absence of clay minerals is an indicator, that the material had been exposed to high temperatures. However minerals, which are formed at high temperatures such as mullite, are also missing. Inarguably, smelting additives were used. The chemical results were confirmed by the mineralogical analysis.

\section{Remarks on the fuel used}

There is a lot of woodland around the site and it is obvious that wood was used as fuel, even though we have no conclusive evidence. Most of the slags show marked wood traces. However, we do not know, if natural wood or charcoal was used. The highways are not favourable to the transportion of mineral coal, so importing is out of the question. The amount of phosphate (P2O5) we measured is not unusual, so firing with Yak dung or bones is improbable. A final definite assessment is not possible.

\section{Remarks to the output}

To calculate the production quantities, we need to know the iron mineral, which was used. Unfortunately we do not have samples. 


\section{Remarks on the iron mineral used}

As already mentioned, we have no information on the iron mineral, which was used. But we may assume, that it was a very rich mineral, because the slags show a 64 $\% \mathrm{Fe} 2 \mathrm{O} 3$ content. Therefore the iron mineral must have contained more than 65 $\% \mathrm{Fe} 2 \mathrm{O} 3$. The mineral is silicium-aluminous and contains some $\mathrm{MgO}$, but very little $\mathrm{CaO}$ and $\mathrm{K} 2 \mathrm{O}$. The $\mathrm{P} 2 \mathrm{O} 3$ content is more than $0.1 \%$, but less than $1 \%$, which indicates a phosphoric mineral. The typical trace elements ( $\mathrm{Cr}, \mathrm{V}, \mathrm{Ni}, \mathrm{Cu}$, etc.) are also represented with very low content. Due to the high relationship of SiO2 to $\mathrm{Al} 2 \mathrm{O}$, minerals like laterite, which originate from continental, exterior weathering can be excluded, as well as oolitic minerals, because of the low $\mathrm{CaO}$ and P2O5 content. Sulphur veins can be excluded in the absence of sulphur evidence as well as banded iron formation because of a too high Al2O3 content Magnetite (SkarnType) cannot be excluded, but there is no obvious evidence such as $\mathrm{Sn}$, Ba, etc. The mining of hematite in a grantic environment is probable.

\section{Conclusion of the ${ }^{14} \mathrm{C}$-dating}

The radiocarbon dating of the samples, which originate from the castle, give dates of $1480-1650 \mathrm{AD}$. This dating is consistent with the ${ }^{14} \mathrm{C}$-analysis results of excavated ceramic samples. However, the charcoals of the smelting place date back to $1165-1265$ AD. We have not yet been able to prove if this time difference is in accordance with the facts or whether approximately 300 year old wood was used to heat the furnace, which would certainly be possible in an area of dense and uncultivated jungle.

\section{Conclusions}

We do not know whether the iron smelting at Nag Lhakhang is related to the wealth of the monastery-castle Drapham Dzong.

The slag sample Na7, which was found on the Drapham-Dzong, could be a calotte,(see my previous query at the beginning) but this is uncertain. The sample is mixed up with sand and other soil materials and does not show the typical structures, which could clearly identify a calotte.

If we assume, that 300 year old wood has been used as fuel in the iron smelting 
furnace, then we could assume a relation between slag $\mathrm{Na} 7$ and the slags from Nag Lhakang. But this has first to be proved.

It can be expected that the samples originate from iron smelting only and that no heavy or precious metals have been smelted.

It is difficult to assess if the iron mineral comes from the region itself. From a strictly geological view point there is good evidence. Capt. R. Boileau Pemberton, a British ambassador, mentioned in his report in 1839 some iron mining at Tassgong (Tongsa): [...] spear and arrow heads are manufactured principally at Tassgong [Tongsa], from iron procured in the hills, at the northern foot of the castle, and large copper cauldrons are formed from the metal, which is said to be obtained in the hills at the foot of Tassangsee, which place is celebrated throughout Bootan [Bhutan] for its superiority in their manufacture. (Boileau Pemberton R., 1839 (Rpr. 2005), p. 77). The distance between Tongsa and Nag Lhakang is about 70 $\mathrm{km}$ (today a 3 hour drive).

From the same source (Boileau Pemberton R., 1839 (Rpr. 2005), p. 79) we learn about the import- and export statistics of the caravans in those days; that silver was imported and copper exported. Iron is absent from both statistics.

The chemico-mineralogical analysis has revealed that most probably a bloomery furnace was used for the iron smelting with a temperature of about $1^{\prime} 250^{\circ} \mathrm{C}$. We assume that wood or charcoal was used as fuel, but this could be not yet proved. How the bloomery furnace was built, we do not know. They used natural stones and the furnace inside walls were covered with loam, as is usual in other parts of the world.

We do not know how the aeration worked. One sample (H100) could be a fragment of an aeration nozzle, due to the slightly convex form and quality of the material. But this is speculative.

We do not know what output was achieved. However, the insufficient reduction of the slag samples and the huge slags of the heap may either indicate that the temperatures were too low, or the furnace was inadequately built or that a very rich mineral was used. In the latter case, despite insufficient reduction, the foundry yield was satisfactory.

Despite the lack of archaeological content, we nevertheless assembled considerable information and observations, which will assist further investigations of the site. It is to be hoped that this will be possible before the relic trade has blurred all traces of this wonderful discovery. 


\section{Prospektionsbericht}

Dominique Oppler

\section{Einführung}

Während der Grabungskampagne 2009 auf dem Drapham-Dzong (Bhutan, Provinz Bumthang), kamen in der Schicht Na3, also innerhalb von Müllablagerungen ausserhalb der Westkurtine, Eisenschlackenfragmente zum Vorschein (Probe Nr. Na7 und N2/8). Zudem fand sich, dank Hinweisen aus der Bevölkerung, eine mächtige Schlackenhalde im Bambusdschungel nahe des Weilers Nag Lhakhang, ca. 1 km Luftlinie nordöstlich des Drapham-Dzongs, resp. ca. 650 m Luftlinie südöstlich vom Weiler entfernt (Abb. 2).

2010 wurde die Schlackenhalde nachprospektiert und das Gebiet rund um die Halde in einem ca. 100 m Radius abgeschritten. Dabei wurden weitere zwei beachtenswerte Fundplätze festgestellt.

In ca. 16 m Entfernung, nordwestlich der Schlackenhalde, fand sich eine auffällige Steinansammlung; im Sinne einer Arbeitshypothese nachfolgend als Ofenplatz bezeichnet. Im Weiteren fiel uns in ca. 50 m nordöstlicher Entfernung des Ofenplatzes eine auffallend flache Plattform, mit einem Durchmesser von ca. $10 \mathrm{~m}$ auf. Eine staatliche Grabungserlaubnis lag nicht vor. Die Landbesitzerin willigte ein, die Fundplätze von dichtestem Bambus und sonstigem Gestrüpp zu säubern, zu vermessen und nur oberflächlich zu beproben.

Der vorliegende Bericht steht daher unter der Prämisse, dass es sich lediglich um Prospektionsresultate handelt, welche erst durch spätere Grabungen verifiziert 
und vertieft werden können. Weil keine moderneren vermessungstechnischen Instrumente zur Verfügung standen, wendeten wir traditionelle Mittel an (Kompass, Klappmeter, Augenmass). Grössere Distanzangaben, Höhen und Koordinaten wurden später über Google-Earth ${ }^{1}$ bestimmt.

Der Zusammenhang zwischen den Schlackenfunden auf dem Drapham-Dzong und jenen der metallurgischen Industriestätte bei Nag Lhakhang, bildet eine der zentralen Fragestellungen dieser Untersuchung. Wenn die Nachforschungen uns nicht getäuscht haben, ist es auch das erste Mal, dass in dieser Zone, an den Hängen des Hoch-Himalajas, metallurgische Industrie nachgewiesen und untersucht werden konnte.

\section{Ökosystem und topographische Anordnung der Fundplätze}

Die Fundorte liegen oberhalb Nag Lhakhang (ca. 2824 m.ü.M.) in einem kleinen Seitental, das bis zu den Fundplätzen, auf ca. 2950 m.ü.M. mit einem Gefälle von ca. $10^{\circ}$ anhebt. Das Tal wird nördlich und südlich von niederen Hügelzügen eingegrenzt, welche sich durch erodiertes Geröll des östlich verlaufenden Bergzuges (bis ca. 4170 m.ü.M.) gebildet haben. Zwei kleinere Wildbäche ziehen entlang dieser Erhebungen und ergiessen sich im Choekhor Toed Tal bei Nag Lhakhang in einen Bach, welcher selbst im Hauptfluss, dem Chamka-Chu, mündet.

Das Tal besteht aus Trockenwiesen die als Weidland genutzt werden. Die humose Schicht (Ranker/Ah-C) bedeckt nur knapp den steinigen bis felsigen Untergrund.

1 Zugriff vom 7.8.2012 


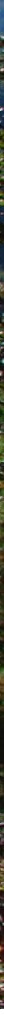

Abb. 1:

Drapham-Dzong im

Choekhor-Tal (Kegel

Bildmitte). Links im

Bild das Areal mit dem

Verhüttungsplatz.

Die Fundplätze befinden sich zwischen dem südlich gelegenen Bachlauf und dem Nordfuss des südlichen Hügelzuges. Das Gebiet dürfte erst in den vergangenen paar Jahren von schnellwachsendem Himalaja-Bambus, Dornengestrüpp und niedrigstämmigen Laubholzbüschen überwachsen worden sein, oder die Bodenstruktur bietet dem Wachstum grösserer Bäume zuwenig Raum. Jedenfalls fällt auf, dass dieser Bereich nur von schwachem Unterholz bewachsen ist und sich daher vom kräftigen, älteren Bewuchs des benachbarten Hügels klar abgrenzt (Abb. 1).

Plattform, Ofenplatz, Schlackenhalde liegen auf einer Ost-West Achse von insgesamt 80 m Länge (westlichste Ausdehnung der Schlackenhalde bis zur östlichsten Begrenzung der Plattform), wobei die Plattform auf dem höchsten Punkt liegt. Der Ofenplatz befindet sich ca. $50 \mathrm{~m}$ weiter unten, ebenfalls auf einem horizontalen Abschnitt des Berghanges. Die Schlackenhalde beginnt ca. $16 \mathrm{~m}$ unterhalb des Ofenplatzes und ergiesst sich über einen vermutlich natürlich gebildeten Abhang (Abb. 5). 


\section{Die Plattform}

Die Plattform ist horizontal zum Hang angelegt und hat einen Durchmesser von ca. 10 m. Westlich, in Richtung Ofenplatz, wird die Plattform von einer steil abfallenden Rampe von ca. 2,5 m Höhe begrenzt.

Die Plattform ist nur von Gräsern und Farnen bewachsen und wird am Fusse des westlichen Abhangs von älteren, stark zerklüfteten immergrünen Eichen eingefriedet.

Die Plattform liegt an einem ca. $10^{\circ}$ geneigten Abhang, ist topfeben und in ihrer Ausdehnung klar begrenzt. Wir gehen daher davon aus, dass es sich um eine Aufschüttung handelt, die anthropogen angelegt wurde.

Unsere Hypothese, dass es sich hier womöglich um einen Köhlerplatz handelt, hat sich ausschliesslich aus der Tatsache entwickelt, dass die Plattform mit den Standorten Ofenplatz und Schlackenhalde auf derselben Achse liegt. Wir haben aber keine Bodenproben nehmen und auch keine Oberflächenfunde machen können, womit die Hypothese nicht weiter untersucht noch bestätigt werden konnte.

\section{Der Ofenplatz ${ }^{1}$}

Die Umgebung des Ofenplatzes war dermassen von Gebüschen, Bambus und Dornengestrüpp überwuchert, dass man nur mit Mühe zur auffälligen Steinansammlung gelang. Als erstes haben wir den Platz über eine Fläche von ca. $7 \times 7$ $m$ freigelegt, resp. vom Gestrüpp befreit. Innerhalb dieser Fläche bedeckten die sichtbaren Erhebungen eine Fläche von ca. 6 × 6 m (Abb. 6 - 8).

Die Steinansammlung machte den Eindruck einer in sich eingestürzten Struktur. Jedenfalls konnten keine zusammenhängenden Baustrukturen festgestellt werden.

Auf der nord-östlichen Seite überragte die Steinansammlung das Gehniveau um ca. $50 \mathrm{~cm}$, in einer Mächtigkeit von ca. $2 \mathrm{~m}$ Breite x $5 \mathrm{~m}$ Länge. In nord-westlicher Richtung, also in Richtung der Schlackenhalde, nimmt die Anzahl der sich überlagernden Steine merklich ab und die Lage derselben verengt sich in der Weise, dass ein Ausfluss (Arbeitshypothese) vermutet werden könnte.

Durch diverse Spalten in der Mitte des oberen Drittels der Steinansammlung massen wir eine Tiefe von mind. $60 \mathrm{~cm}$, was eine Grube vermuten lässt, in welche 

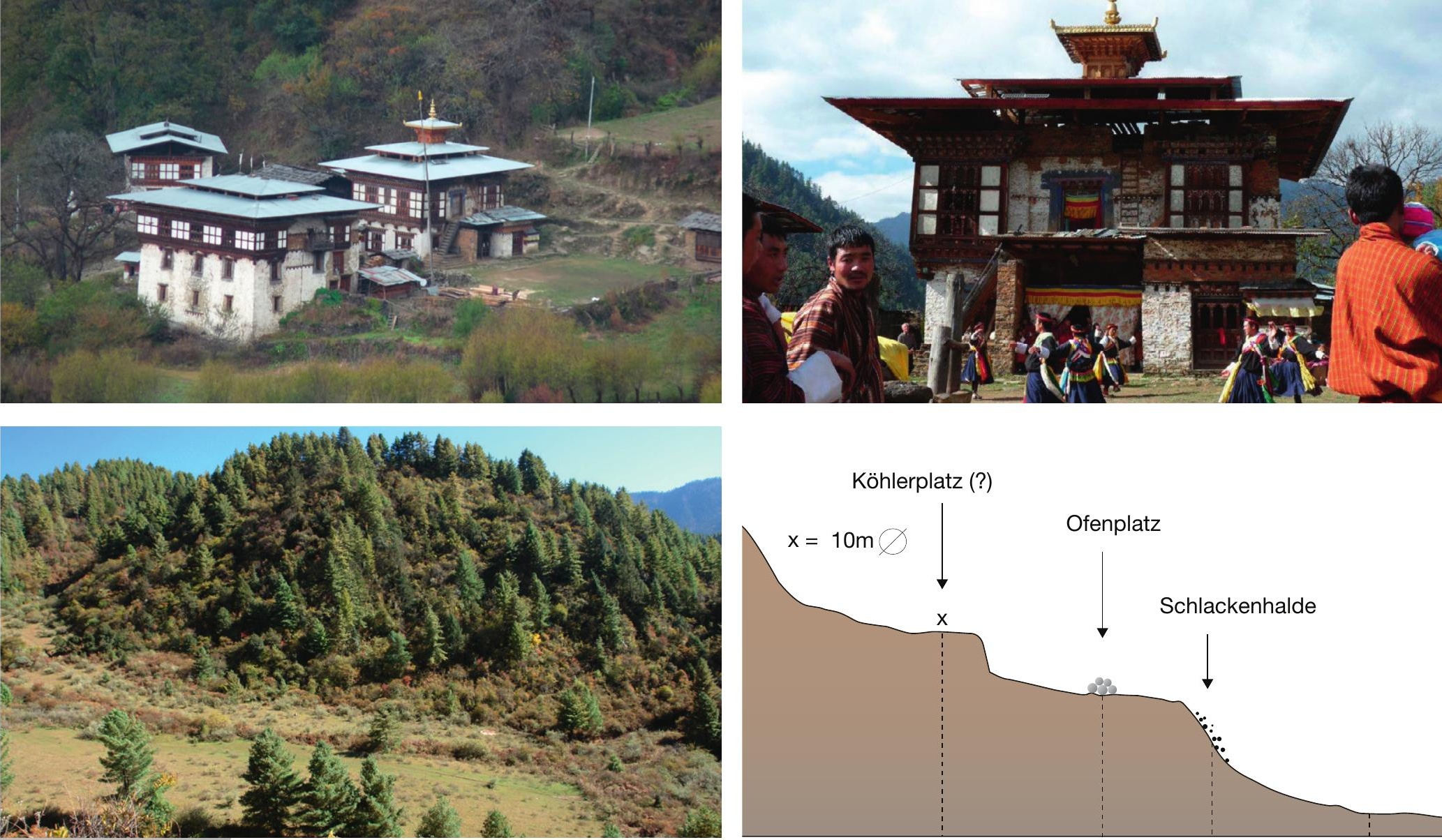

Abb. 2:

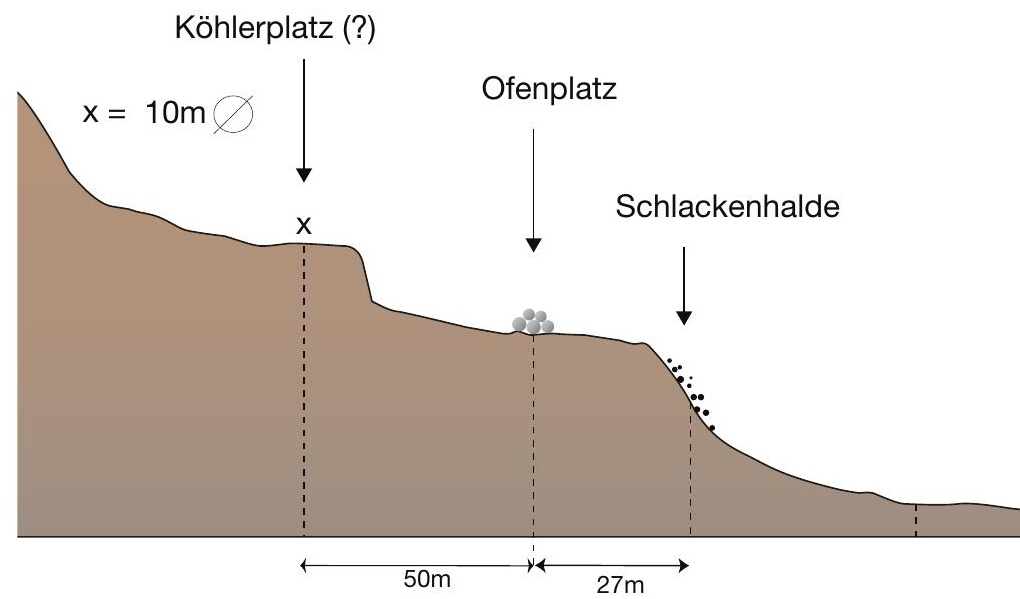

Nag Lhakhang,

Kloster und Gehöft.

Abb. 3:

Das Kloster von

Nag Lhakang während

eines religiösen

Festes.

Abb. $4+5$ :

Areal mit Plattform, Ofenplatz und Schlakkenhalde. Daneben: topografisches

Schema (stark überzeichnet). die Steine eingebrochen sein dürften.

Es handelt sich mehrheitlich um granitisches, naturbelassenes Gestein in unterschiedlichsten Massen (bis ca. 50 × 70 cm). Vereinzelte Bearbeitungsspuren können nicht ausgeschlossen werden. Im mittleren Bereich der Gesteinsansammlung, wie auch im Bereich des Ausflusses fanden sich rötlich verfärbte Steine.

Im unteren Drittel des Ausflussbereiches lasen wir vereinzelt kleine Schlackenfragmente auf, welche auf dem Gehhorizont oberflächlich verteilt waren. Reichlich angehäuftes Schlackenvorkommen, von nur wenig Humus überdeckt, konnte beidseits, wie auch in der Verlängerung des Ausflusses festgestellt werden. Zudem wurde an der nördlichen Aussenseite des Ausflusses ein Ofenwandfragment gefunden (Probe Nr. 0201).

Es wurden diverse Schlackenproben entnommen deren Analyse weiter unten besprochen wird (s. Probeninventar, Signatur „O“). 


\section{Schlackenhalde}

Die Schlackenhalde, welche sich ca. $16 \mathrm{~m}$ unterhalb des Ofenplatzes befindet, quillt aus humoser Schicht hervor, am Abbruch einer erhöhten Plattform, und bildet eine Halde von ca. 30 m Länge in südwestlicher Richtung. Die seitliche Begrenzung konnte aufgrund starken Bewuchses und humoser Bedeckung nicht festgestellt werden. Die Schlacken liegen lediglich ca. 4 - 5 m unbedeckt am Hang, die Halde dürfte aber eine Mächtigkeit von mindestens 6 - $8 \mathrm{~m}$ Breite aufweisen. Auch konnte nicht festgestellt werden, wie weit sich das Schlackenvorkommen auf dem oberhalb liegenden Terrain in Richtung Ofenplatz erstreckt. Jedenfalls konnten innerhalb der Distanz von ca. $16 \mathrm{~m}$ zwischen Ofenplatz und Ansatz der Halde, zahlreiche Schlacken bereits auf Gehniveau und in humoser Schicht festgestellt werden.

In der Halde liegen auffällig viele, besonders mächtige Schlacken, schwer, stark oxidiert, vom Typus GDS ${ }^{1}$, ohne markante Fliessformationen. Sie weisen durchschnittlich Grössen von ca. $20-30 \mathrm{~cm}$ auf. Die Schlacken tragen meist deutliche Abdrucke von Holz- oder Holzkohle. Mangels Waage konnten keine Gewichte eruiert werden und mit Rücksicht auf erhöhte Flugfrachtkosten, musste sich die Beprobung auf die kleineren Exemplare beschränken (s. Probeninventar, Signatur „H“).

Ähnlich wie beim Ofenplatz auch, befanden sich unter den kleineren Schlackenfragmenten wiederum GDS, teilweise auch mit deutlichen Fliessspuren, wie auch glasige, stark poröse, weisslich bis weiss-graue Schlacken vom Typus GPS ${ }^{2}$. Auch konnte gebrannter, teilweise auch verschlackter Ton beprobt werden. Kalotten konnten keine ausgemacht werden.

Innerhalb der Schlackenhalde befinden sich zahlreiche Natursteinbrocken in ebensolchem Format und Gestein wie bereits für den Ofenplatz beschrieben.

Sie weisen teilweise Erhitzungs- und Verschlackungsspuren auf (Abb. 11 + 12). Es ist davon auszugehen, dass das Schlackenvolumen der Halde den tatsächlichen Produktionsabfällen nicht mehr entspricht. Wir haben nämlich festgestellt, dass die Schlacken der Bevölkerung viel bedeuten.

Nachfragen bei bhutanischen Arbeitern der Drapham Dzong-Grabung haben ergeben, dass die metallurgische Industrie an jenem Ort der Sage nach auf Lama Terton Pema Lingpa ${ }^{3}$ zurückgeht. Dieser Lama gilt heute bei den Gläubigen als heilig. Die Schlacken werden daher als Reliquien gesammelt und gehandelt.

\footnotetext{
1 Graue-dichte-Schlacke.

2 Graue-poröse-Schlacke..

3 Pema Lingpa (1450 - 1521) [je nach Region auch Padma Gling-pa genannt] ist einer der meist verehrten Lamas in Bhutan. Er wird als Reinkarnation des Guru Rinpoche verehrt. Lama Lingpa wurde in Bumthang geboren und lernte bei seinem Grossvater das Schmieden. (Mayhew B., 2011, S. 175)
} 
- = Schlacke, an der Oberfläche liegend.

$\mathbf{X}=$ Natursteine, unbearbeitet, mit Erhitzungsspuren (rötlich). = Natursteine, unbearbeitet.

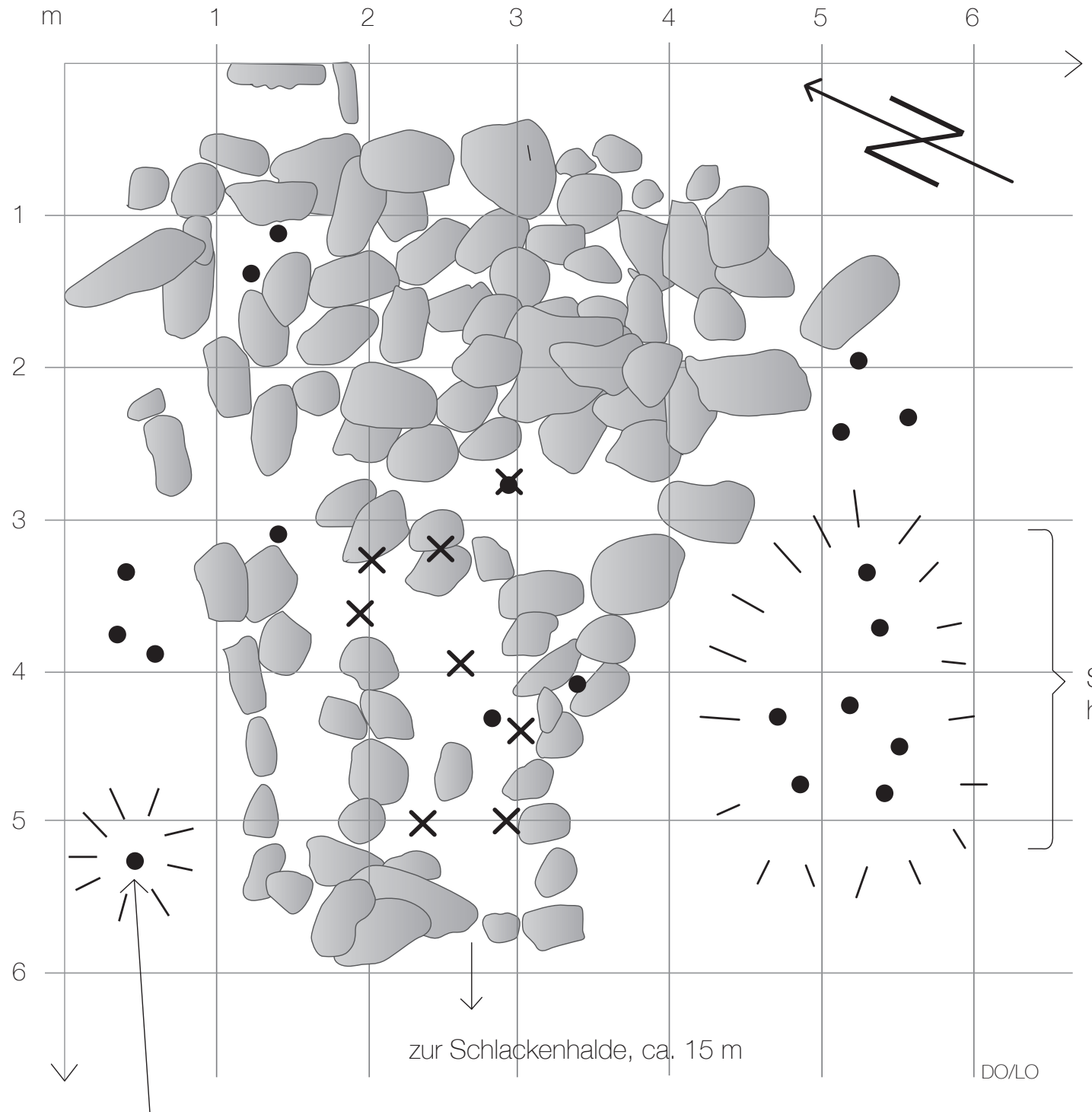

Schlacken-

häufung

Abb. 8:

Situation beim Ofenplatz. Oberflächliche Stein und Schlakkenansammlungen. Zeichnung stark schematisiert

Schlackenhäufung

+ Ofenwand

Höhe: $\quad$ 2'938,2 m.ü.M.

Pos: $\quad \quad \quad \quad 27^{\circ} 39.69800^{\prime}(B$.

E $90^{\circ} 45.61500^{\prime}$ (L.) 


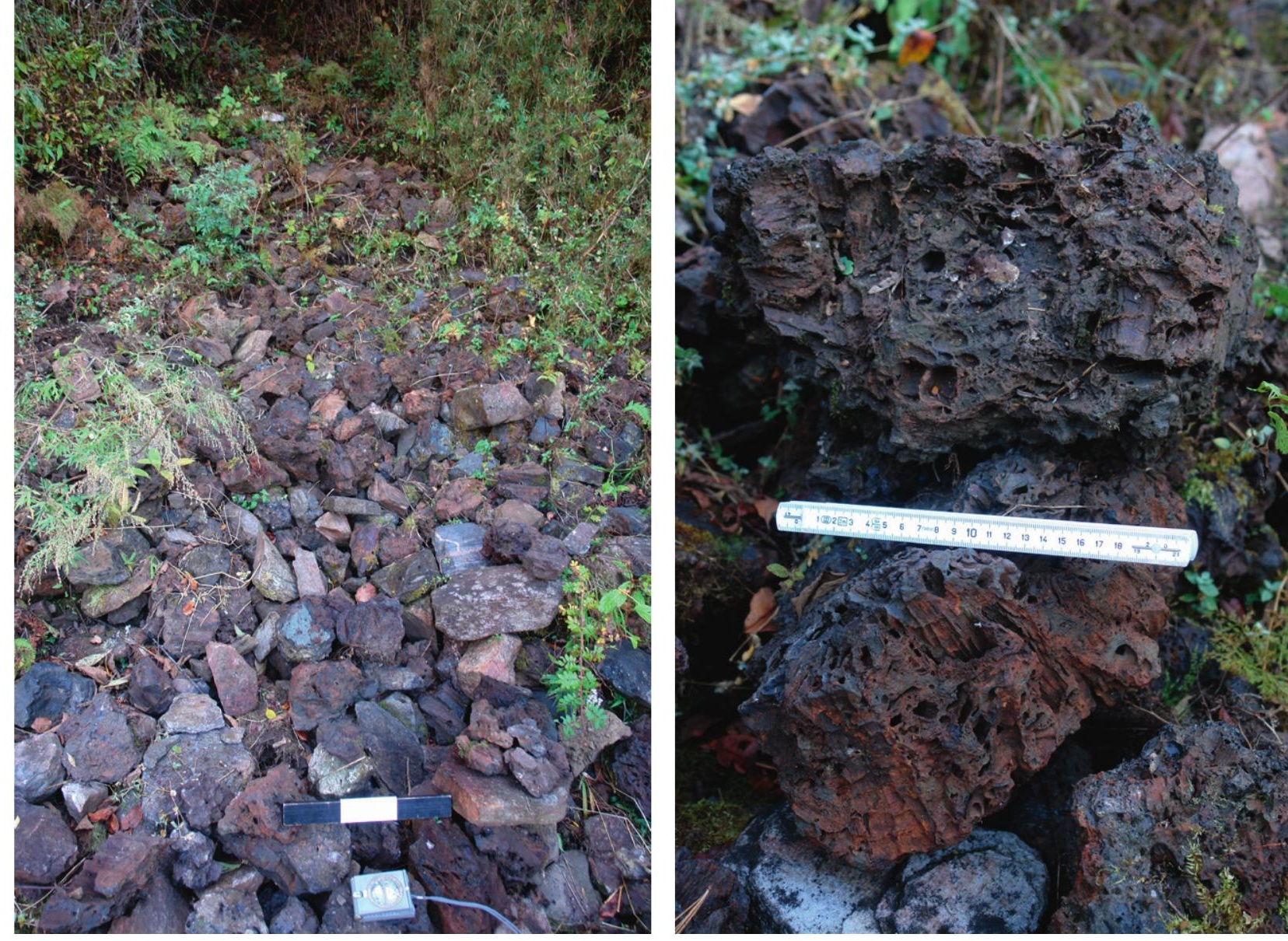

Abb. 9:

Schlackenhalde,

Richtung Nord-Osten

aufgenommen.

Abb. 10:

Dichte, stark

oxidierende Schlacken.

\section{Weitere Erkundungen}

In der Hoffnung, im selben Seitental weitere Verarbeitungsplätze auszumachen, haben wir Bachläufe nach Schlacken und Strukturen abgesucht. Aufgrund des dichten Buschwaldbewuchses war dies allerdings nur partiell möglich. Der Befund fiel negativ aus. 


\section{Analyse der Schlackenproben}

Vincent Serneels, Raphaëlle Soulignac, Dominique Oppler

\section{Vorgehen}

Es wurden insgesamt 58 Proben $(7,5 \mathrm{~kg})$ entnommen, wovon 11 vom Ofenplatz, 43 von der Schlackenhalde und 2 vom Grabungsplatz Drapham-Dzong stammen. Alle Proben wurden gereinigt, fotografiert, vermessen, gewogen, ihre Dichte bestimmt, makroskopisch beschrieben und grob typologisiert.

Von diesem Material wurden 24 Proben einer vertieften Analyse unterzogen, hiervon 5 Proben des Ofenplatzes $(\mathrm{O}), 17$ Proben der Schlackenhalde $(\mathrm{H})$ und 2 Proben des Grabungsplatzes ( $\mathrm{Na})$.

Die 24 Proben wurden gezeichnet, die Schnittlage bestimmt und mit dem Diamantschneider halbiert, die Schnittflächen anschliessend fotografiert und nachgezeichnet.

Von 19 dieser zersägten Proben, wurde je eine Hälfte gemahlen und sowohl der XRD'-Analyse (mineralogische Untersuchung), wie auch der $\mathrm{XRF}^{2}$-Analyse (elementare Untersuchung) unterzogen.

Bei der chemischen Analyse wurden Haupt- und Spurenelemente gemessen. Der Materialverlust während der Feuerung wurde nicht gemessen.

Alle gemessenen Materialien weisen Fusionsspuren bei hohen Temperaturen auf. Es konnten keine signifikanten Oxydations- oder Hydratationsspuren nachgewiesen werden. Bei diesen Verhältnissen wäre die Bestimmung des Materialver-

$\begin{array}{ll}1 & \text { X-ray Diffraction } \\ 2 & \text { X-ray Fluorescence }\end{array}$ 
lustes ein Indikator um auf das vorhandenen $\mathrm{FeO}$ zu schliessen, wir haben aber von diesen Untersuchungen vorerst abgesehen.

Aufgrund der chemischen und mineralogischen Zusammensetzungen wurden 3 Gruppen gebildet und von diesen je ein Dünnschliff angefertigt. Die Schliffe wurden unter dem optischen Mikroskop beschrieben und fotografiert.

Die Analysen erfolgten bei Geoscience der Universität Fribourg (Schweiz) unter Supervision von Prof. Dr. Vincent Serneels, in Zusammenarbeit mit Raphaëlle Soulignac und Dominique Oppler. Die Dünnschliffe wurden von Jean-Pierre Bourqui gefertigt.

Beim zersägen der Schlackenprobe H107a wurden Holzkohlestückchen entdeckt. Ebenso hafteten an der Probe Na7, welche vom Grabungsplatz stammt, feine Holzkohlenflitter. Die Holzkohlen wurden im C14-Labor der ETH Zürich datiert. Aufbereitung, Analyse und Interpretation wurden mit Hilfe von Dr. Irka Hajdas durch Dominique Oppler ausgeführt. (Tab. 1)

\section{Bemerkungen zur regionalen Geologie}

Im Rahmen der Grabungskampagne war es nicht möglich in der Region des Drapham-Dzong nach potentiellen Abbaustellen des Eisenminerals zu suchen und es gibt noch sehr wenige Angaben zu dieser Frage.

Generell kann festgehalten werden, dass die Fundstelle sich auf dem granitischen Sockel des Hoch-Himalajas befindet, welcher die Bildung klassischer Eisenminerale wenig begünstigt. Man kann daher Minerale, welche aus Verwitterungsprozessen stammen ausschliessen (Laterit im weitesten Sinne). Auch kann marines, sedimentäres, oolitisches Mineral ausgeschlossen werden. Hingegen dürften magmatische oder metamorphe Minerale erwartet werden, wie z.B. schwefelhaltige Adern ${ }^{3}$, Hämatit oder Magnetit. Für in Kontaktmetamorphose gebildetem Magnetit (magnetitischer Skarn) könnten die Bedingungen günstig sein.

3 die jedoch zur Verhüttung eher ungünstig sind. 
Tab. 1:

Übersicht der

Proben und ange-

wandten Untersuch-

ungsmethoden.

\section{Makroskopische Klassifikation}

Auf Basis makroskopischer Beschreibung, können 3 Materialgruppen unterschieden werden:

- Graue-dichte-Schlacken (GDS), fayalitisch, teilweise mit hohem Eisenanteil.

- $\quad$ Glasige Schlacken (GPS), die einen sind hell(GPSh), andere sind dunkel (GPSd).

- Lehmig, sandiges Material, mehr oder weniger geschmolzen, teilweise mit Felseinschlüssen. Es handelt sich um Wandfragmente (eventuell um Düsenfragmente) welche hohen Temperaturen ausgesetzt waren und den Schmelzpunkt erreicht haben. 


\section{Resultate der Probenuntersuchungen}

\begin{tabular}{|c|c|c|c|c|c|c|c|}
\hline & & \multicolumn{4}{|c|}{ Makroskopie } & \multirow{2}{*}{\begin{tabular}{|l} 
Hauptelemente \\
XRF \\
\end{tabular}} & \multirow{2}{*}{\begin{tabular}{|l|}
$>1000$ \\
Counts \\
XRD
\end{tabular}} \\
\hline Typ & $\begin{array}{l}\text { Proben- } \\
\text { Nr. }\end{array}$ & Fotografie & Schnitt & \begin{tabular}{|l|} 
Masse \\
Länge x Breite x \\
Dicke
\end{tabular} & Gewicht & & \\
\hline \multirow[t]{8}{*}{ GDS } & O206a & & & $\begin{array}{l}99,5 \times 59,0 \times 39,0 \\
\mathrm{~mm}\end{array}$ & $260 \mathrm{~g}$ & $\begin{array}{l}\mathrm{Fe} 2 \mathrm{O} 3 / 64.5 \% \\
\mathrm{SiO} / 20.6 \% \\
\mathrm{Al} 2 \mathrm{O} 3 / 7.8 \%\end{array}$ & \begin{tabular}{|l} 
Fayalit/ \\
Wüstit
\end{tabular} \\
\hline & O206b & & & $\begin{array}{l}85,0 \times 61,0 \times 38,0 \\
\mathrm{~mm}\end{array}$ & $170 \mathrm{~g}$ & $\begin{array}{l}\mathrm{Fe} 2 \mathrm{O} 3 / 59.1 \% \\
\mathrm{SiO} 2 / 23.8 \% \\
\mathrm{Al} 2 \mathrm{O} 3 / 9.0 \% \\
\mathrm{~K} 2 \mathrm{O} / 2.0 \%\end{array}$ & $\begin{array}{l}\text { Fayalit/ } \\
\text { Wüstit }\end{array}$ \\
\hline & $\mathrm{H} 45 \mathrm{a}$ & & & $\begin{array}{l}100,5 \times 59,0 \times 51,5 \\
m m\end{array}$ & $460 \mathrm{~g}$ & $\begin{array}{l}\mathrm{Fe} 2 \mathrm{O} 3 / 71.9 \% \\
\mathrm{SiO} 2 / 16.1 \% \\
\mathrm{Al} 2 \mathrm{O} 3 / 4.8 \%\end{array}$ & $\begin{array}{l}\text { Fayalit/ } \\
\text { Wüstit }\end{array}$ \\
\hline & $\mathrm{H} 45 \mathrm{~b}$ & & & $\begin{array}{l}110,3 \times 70,0 \times 33,0 \\
\mathrm{~mm}\end{array}$ & $280 \mathrm{~g}$ & $\begin{array}{l}\mathrm{Fe} 2 \mathrm{O} 3 / 56.6 \% \\
\mathrm{SiO} 2 / 25.4 \% \\
\mathrm{Al} 2 \mathrm{O} 3 / 9.5 \%\end{array}$ & Fayalit \\
\hline & $\mathrm{H} 107 \mathrm{a}$ & & & $\begin{array}{l}100,5 \times 59,0 \times 51,5 \\
\mathrm{~mm}\end{array}$ & $460 \mathrm{~g}$ & $\begin{array}{l}\mathrm{Fe} 2 \mathrm{O} 3 / 63.7 \% \\
\mathrm{SiO} 2 / 20.6 \% \\
\mathrm{Al} 2 \mathrm{O} 3 / 8,2 \%\end{array}$ & $\begin{array}{l}\text { Fayalit/ } \\
\text { Wüstit }\end{array}$ \\
\hline & $\mathrm{H} 108$ & & & $\begin{array}{l}115,5 \times 81,0 \times 56,0 \\
\mathrm{~mm}\end{array}$ & $400 \mathrm{~g}$ & $\begin{array}{l}\mathrm{Fe} 2 \mathrm{O} 3 / 63.9 \% \\
\mathrm{SiO} 2 / 20,5 \% \\
\mathrm{Al} 2 \mathrm{O} 3 / 8,4 \%\end{array}$ & $\begin{array}{l}\text { Fayalit/ } \\
\text { Wüstit }\end{array}$ \\
\hline & $\mathrm{H} 115 \mathrm{a}$ & & & $\begin{array}{l}88,0 \times 69,0 \times 46,0 \\
\mathrm{~mm}\end{array}$ & $250 \mathrm{~g}$ & $\begin{array}{l}\mathrm{Fe} 2 \mathrm{O} 3 / 67.5 \% \\
\mathrm{SiO} 2 / 21.1 \% \\
\mathrm{Al} 2 \mathrm{O} 3 / 6,8 \%\end{array}$ & $\begin{array}{l}\text { Fayalit/ } \\
\text { Fe3O4 }\end{array}$ \\
\hline & H116a & & & $\begin{array}{l}93,0 \times 72,0 \times 41,0 \\
\mathrm{~mm}\end{array}$ & $185 \mathrm{~g}$ & $\begin{array}{l}\mathrm{Fe} 2 \mathrm{O} 3 / 11.8 \% \\
\mathrm{SiO} 2 / 63.3 \% \\
\mathrm{Al} 2 \mathrm{O} 3 / 17.7 \% \\
\mathrm{~K} 2 \mathrm{O} / 2.9 \%\end{array}$ & $\begin{array}{l}\text { Quartz } \\
\mathrm{SiO} 2\end{array}$ \\
\hline
\end{tabular}




\begin{tabular}{|c|c|c|c|c|c|c|c|}
\hline & & \multicolumn{4}{|c|}{ Makroskopie } & \multirow{2}{*}{$\begin{array}{l}\text { Hauptelemente } \\
\text { XRF }\end{array}$} & \multirow{2}{*}{$\begin{array}{l}>1000 \\
\text { Counts } \\
\text { XRD }\end{array}$} \\
\hline Tyр & $\begin{array}{l}\text { Proben- } \\
\text { Nr. }\end{array}$ & Fotografie & Schnitt & \begin{tabular}{|l} 
Masse \\
Länge x Breite x \\
Dicke
\end{tabular} & Gewicht & & \\
\hline \multirow[t]{10}{*}{ GPS } & $\mathrm{O} 203$ & & & $84,5 \times 57,0 \times 37,0 \mathrm{~mm}$ & $110 \mathrm{~g}$ & $\begin{array}{l}\mathrm{Fe} 2 \mathrm{O} 3 / 7.4 \% \\
\mathrm{SiO} 2 / 68.6 \% \\
\mathrm{Al} 2 \mathrm{O} 3 / 17.8 \% \\
\mathrm{~K} 2 \mathrm{O} / 3.1 \%\end{array}$ & $\begin{array}{l}\text { Quartz } \\
\mathrm{SiO2}\end{array}$ \\
\hline & O204 & & & $\begin{array}{l}66,5 \times 49,0 \times 39,0 \\
\mathrm{~mm}\end{array}$ & $100 \mathrm{~g}$ & $\begin{array}{l}\mathrm{Fe} 2 \mathrm{O} 3 / 10.3 \% \\
\mathrm{SiO} 2 / 64.6 \% \\
\mathrm{Al} 2 \mathrm{O} 3 / 16.7 \% \\
\mathrm{~K} 2 \mathrm{O} / 4.1 \%\end{array}$ & $\begin{array}{l}\text { Quartz } \\
\mathrm{SiO} 2\end{array}$ \\
\hline & $\mathrm{H} 104 \mathrm{a}$ & & & $81,0 \times 45,5 \times 42,0 \mathrm{~mm}$ & $90 \mathrm{~g}$ & $\begin{array}{l}\mathrm{Fe} 2 \mathrm{O} 3 / 6.0 \% \\
\mathrm{SiO} 2 / 69.0 \% \\
\mathrm{Al} 2 \mathrm{O} 3 / 18.2 \% \\
\mathrm{~K} 2 \mathrm{O} / 3.1 \%\end{array}$ & $\begin{array}{l}\text { Quartz } \\
\mathrm{SiO} 2\end{array}$ \\
\hline & $\mathrm{H} 104 \mathrm{e}$ & & & $87,0 \times 78,0 \times 58,0 \mathrm{~mm}$ & $130 \mathrm{~g}$ & $\begin{array}{l}\mathrm{Fe} 2 \mathrm{O} 3 / 4.2 \% \\
\mathrm{SiO} 2 / 70.5 \% \\
\mathrm{Al} 2 \mathrm{O} 3 / 18.8 \% \\
\mathrm{~K} 2 \mathrm{O} / 3.0 \%\end{array}$ & $\begin{array}{l}\text { Quartz } \\
\mathrm{SiO} 2 / \\
\text { Mullit }\end{array}$ \\
\hline & $\mathrm{H} 104 \mathrm{f}$ & & & $\begin{array}{l}83,0 \times 60,0 \times 43,0 \\
\mathrm{~mm}\end{array}$ & $140 \mathrm{~g}$ & $\begin{array}{l}\mathrm{Fe} 2 \mathrm{O} 3 / 6.8 \% \\
\mathrm{SiO} 2 / 68.5 \% \\
\mathrm{Al} 2 \mathrm{O} 3 / 17.7 \% \\
\mathrm{~K} 2 \mathrm{O} / 3.2 \%\end{array}$ & $\begin{array}{l}\text { Quartz } \\
\mathrm{SiO} 2\end{array}$ \\
\hline & $\mathrm{H} 104 \mathrm{~g}$ & & & $63,0 \times 49,0 \times 31,0 \mathrm{~mm}$ & $80 \mathrm{~g}$ & $\begin{array}{l}\mathrm{Fe} 2 \mathrm{O} 3 / 5.6 \% \\
\mathrm{SiO} 2 / 64.5 \% \\
\mathrm{Al} 2 \mathrm{O} 3 / 21.5 \% \\
\mathrm{~K} 2 \mathrm{O} / 3.2 \% \\
\mathrm{MgO} / 2.2 \%\end{array}$ & $\begin{array}{l}\text { Quartz } \\
\mathrm{SiO} 2 / \\
\text { Mullit }\end{array}$ \\
\hline & H105 & & & $\begin{array}{l}98,0 \times 93,0 \times 48,0 \\
\mathrm{~mm}\end{array}$ & $210 \mathrm{~g}$ & $\begin{array}{l}\mathrm{Fe} 2 \mathrm{O} 3 / 6.0 \% \\
\mathrm{SiO} 2 / 67.6 \% \\
\mathrm{Al} 2 \mathrm{O} 3 / 17.7 \% \\
\mathrm{~K} 2 \mathrm{O} / 3.8 \%\end{array}$ & $\begin{array}{l}\text { Quartz } \\
\mathrm{SiO} 2\end{array}$ \\
\hline & $\mathrm{H} 117$ & & & $92,0 \times 75,0 \times 61,0 \mathrm{~mm}$ & $310 \mathrm{~g}$ & & \\
\hline & $\mathrm{H} 114 \mathrm{a}$ & & & $\begin{array}{l}100,5 \times 100,0 \times 60,0 \\
\mathrm{~mm}\end{array}$ & $290 \mathrm{~g}$ & $\begin{array}{l}\mathrm{Fe} 2 \mathrm{O} 3 / 6.7 \% \\
\mathrm{SiO} 2 / 64.6 \% \\
\mathrm{Al} 2 \mathrm{O} 3 / 20.4 \% \\
\mathrm{~K} 2 \mathrm{O} / 3.9 \%\end{array}$ & $\begin{array}{l}\text { Quartz } \\
\mathrm{SiO} 2 / \\
\text { Mullit }\end{array}$ \\
\hline & $\mathrm{H} 114 \mathrm{C}$ & & & $\begin{array}{l}74,0 \times 53,0 \times 43,0 \\
m m\end{array}$ & $180 \mathrm{~g}$ & $\begin{array}{l}\mathrm{Fe} 2 \mathrm{O} 3 / 8.8 \% \\
\mathrm{SiO} 2 / 64.3 \% \\
\mathrm{Al} 2 \mathrm{O} 3 / 18.1 \% \\
\mathrm{~K} 2 \mathrm{O} / 3.5 \%\end{array}$ & $\begin{array}{l}\text { Quartz } \\
\mathrm{SiO} 2\end{array}$ \\
\hline
\end{tabular}




\begin{tabular}{|c|c|c|c|c|c|c|c|}
\hline \multirow[b]{2}{*}{ Typ } & \multirow[b]{2}{*}{$\begin{array}{l}\text { Proben- } \\
\text { Nr. }\end{array}$} & \multicolumn{4}{|c|}{ Makroskopie } & \multirow{2}{*}{\begin{tabular}{|l} 
Hauptelemente \\
XRF \\
\end{tabular}} & \multirow{2}{*}{\begin{tabular}{|l}
$>1000$ \\
Counts \\
XRD
\end{tabular}} \\
\hline & & Fotografie & Schnitt & $\begin{array}{l}\text { Masse } \\
\text { Länge x Breite x } \\
\text { Dicke }\end{array}$ & Gewicht & & \\
\hline \multirow[t]{6}{*}{$\begin{array}{l}\text { Tonig-sandige } \\
\text { Proben }\end{array}$} & O201 & & & $\begin{array}{l}99,0 \times 84,5 \times 42,5 \\
\mathrm{~mm}\end{array}$ & n.g. & & \\
\hline & $\mathrm{H} 100$ & & & $\begin{array}{l}86,5 \times 65,0 \times 35,0 \\
\mathrm{~mm}\end{array}$ & n.g. & $\begin{array}{l}\mathrm{Fe} 2 \mathrm{O} 3 / 4.9 \% \\
\mathrm{SiO} 2 / 65.6 \% \\
\mathrm{Al} 2 \mathrm{O} 3 / 22.8 \% \\
\mathrm{~K} 2 \mathrm{O} / 2.8 \%\end{array}$ & $\begin{array}{l}\text { Quartz } \\
\text { SiO2 }\end{array}$ \\
\hline & $\mathrm{H} 102$ & & & $\begin{array}{l}93,0 \times 68,0 \times 36,0 \\
\mathrm{~mm}\end{array}$ & n.g. & $\begin{array}{l}\mathrm{Fe} 2 \mathrm{O} 3 / 8.8 \% \\
\mathrm{SiO} 2 / 64.9 \% \\
\mathrm{Al} 2 \mathrm{O} 3 / 20.0 \% \\
\mathrm{~K} 2 \mathrm{O} / 2.6 \%\end{array}$ & $\begin{array}{l}\text { Quartz } \\
\text { SiO2 }\end{array}$ \\
\hline & $\mathrm{H} 112$ & & & $\begin{array}{l}98,0 \times 86,0 \times 40,0 \\
\mathrm{~mm}\end{array}$ & n.g. & & \\
\hline & $\mathrm{Na} 7$ & & & $\begin{array}{l}120,8 \times 94,0 \times 50,0 \\
m m\end{array}$ & $550 \mathrm{~g}$ & & \\
\hline & $\mathrm{N} 2 / 8$ & & & $56,6 \times 34,5 \times 17,0 \mathrm{~mm}$ & $30 \mathrm{~g}$ & & \\
\hline
\end{tabular}




\section{Die XRD-Analyse - mineralogische Untersuchung}

\section{Die GDS}

Die 7 analysierten Proben beinhalten alle einen hohen Anteil Fayalit $\left(\mathrm{Fe}_{2} \mathrm{SiO}_{4}\right)$, das Eisensilikat, welches gewöhnlich in stark eisenhaltigen Schlacken vorkommt. Fayalit ist mit einem Oxyd der Familie der Spinelle vergesellschaftet, welches reich an Aluminium ist. Die Zusammensetzung dieser Spinelle scheint etwas veränderlich zu sein. In gewissen Proben ist es Hercynit welches angezeigt wird $\left(\mathrm{Al}_{2} \mathrm{FeO}_{4}\right)$, oder auch das Kaliumaluminiumsilikat, Leucit $\left(\mathrm{KAISiO}_{6}\right)$.

In 6 von 7 Proben sind Eisenoxyde nachgewiesen. Man findet die Minerale Hämatit $\left(\mathrm{Fe}_{2} \mathrm{O}_{3}\right)(3 \mathrm{x})$, Magnetit $\left(\mathrm{Fe}_{3} \mathrm{O}_{4}\right)(3 \mathrm{x})$ und Wüstit $(\mathrm{FeO})(3 \mathrm{x})$. Die Koexistenz dieser drei Minerale wird in metallurgischen Abfällen oft beobachtet. Sie ist auf die hohe Variabilität der Reduktionsvoraussetzungen im Ofen, vor allem auch während der Abkühlungsphase, zurückzuführen. Wenn die Abkühlung in Verbindung mit Sauerstoff erfolgt, kann sich an der Oberfläche Magnetit und Hämatit bilden.

Obschon das Eisen im Dünnschliff makroskopisch sichtbar ist, wurde es nicht nachgewiesen. Die Proportionen sind zu schwach.

Quarz $\left(\mathrm{SiO}_{2}\right)$ wird in vier Proben schwach angezeigt. Aufgrund der Präsenz von Leucit in allen Proben, dürfte dieser Quarzanteil keinen Einfluss auf die Verflüssigung gehabt haben. Es kann angenommen werden, dass diese Körner als solide Partikel sekundärer Herkunft sind, sei es dass sie vom Ofenboden, in Kontakt mit der Ofenwand, oder vom externen Boden, während des Ausflusses aufgenommen wurden. Jedenfalls haben sie keine Fusion durchgemacht.

Tab. 2: Übersicht der Anaylseresultate
Die Verbindung von Fayalit, Wüstit und Leucit lässt Verhüttungstemperaturen von $1250^{\circ} \mathrm{C}$ annehmen. Diese mineralogische Verbindung ist üblich bei Schlacken der Eisenverhüttung. Der hohe Anteil an Eisenoxyden weist auf eine unvollständige, schwache Reduktion. 


\section{Die GPS}

Der hohe Anteil amorphen Materials, weist auf einen Schmelzprozess in hohen Temperaturen.

Alle Proben verfügen über einen hohen Quarzanteil. Durchwegs ist Mullit vorhanden. Die gemessenen Spitzen sind allerdings schwach. Entweder ist die gemessene Menge gering oder die Körner haben schlecht kristallisiert. Mullit ist ein Aluminiumsilikat hoher Temperaturen $\left(>1100^{\circ} \mathrm{C}\right)$.

In einer Probe ist die Präsenz von Cristobalit nachgewiesen, ein polymorphes, hochtemperatur-modifiziertes Siliziumdioxyd $\left(\mathrm{SiO}_{2}\right)$.

In drei Proben wird Indialit identifiziert. Es handelt sich um ein seltenes Mineral, einem Aluminium-/Magnesiumsilikat, das sich bei hohen Temperaturen mit der Reduktion bildet. Die Präsenz dieses Minerals muss jedoch noch bestätigt werden, um weitere Informationen zu den gefahrenen Temperaturen zu liefern'1.

Eisenoxyde wurden in 4 Proben nachgewiesen. In drei Proben waren Hämatit und in einer Probe Magnetit identifiziert worden.

Die Spektren der GPSh und GPSd unterscheiden sich nicht signifikant. Es gibt kein Mineral, das eine systematische Präsenz in der einen oder anderen Klasse markiert. Die drei Proben, welche Indialit beinhalten, gehören alle zur Klasse der GPSd, das Mineral konnte aber nicht in allen GPSd nachgewiesen werden. Dagegen wurde in allen GPSd-Proben Eisenoxyd festgestellt. Allerdings scheinen diese Unterschiede nicht signifikant genug zu sein, um die makroskopischen Farbvarianten der GPS-Klasse zu erklären. Die Untergliederung der GPS in Farbklassen ist zudem erschwert, weil es auch Proben gibt, welche helle und dunkle Partien aufweisen.

\section{Die Tone}

In beiden Proben wurde ein hoher Quarzanteil festgestellt und ein schwacher Anteil Mikroklin der Feldspatfamilie. Beide Proben beinhalten Hämatit, wobei die

\footnotetext{
1 Wir konnten Indialit in drei Spektren nachweisen, was allerdings nochmals durch eine vertiefte Analyse bestätigt werden müsste. Das Indialit ist ein Polymorph des Cordierit. Dieses seltene Mineral wurde als Rekristallisationsprodukt nach der Fusion von sedimentärem Felsgestein (Arkose, Grauwacke) identifiziert, welches durch ein unterirdisches Steinkohlefeuer in Bakaro, Süd-West von Hazaribagh, Bihar, Indien, ausgebrochen ist. (Mikishiro, 1957, Mineral Data Publishing) In einem metallurgischen Ofen können ähnliche Temperatur- und Kohlenmonoxydverhältnisse erreicht werden. Dies könnte daher durchaus mit der Beobachtung kohärent sein. Nach unseren Kenntnissen wurde Indialit bisher nicht im Rahmen metallurgischer Abfallprodukte beschrieben.
} 
gemessene Spitze in Probe H102 (rot) ist deutlich höher als jene der Probe H100 (weisslich).

Die Absenz von Tonmineralen ist ein Indikator, dass das Material hohen Temperaturen ausgesetzt war. Allerdings fehlen aber auch die Minerale, welche sich in hohen Temperaturen bilden, wie Mullit, etc.

\section{Chemische Zusammensetzung der Proben}

\section{Vergleich GDS - GPS}

Die Präsenz der beiden Schlackentypen GDS und GPS auf demselben metallurgischen Produktionsplatz kann verschiedene Ursachen haben:

Diese beiden Abfallprodukte können von unterschiedlichen Produktionsprozessen stammen:

- Zwei unterschiedliche Metallurgien, Eisen- und Kupferverhüttung, am selben Ort.

- Zwei unterschiedliche Produktionsschritte, Verhüttung und Schmiede, in derselben Produktionskette.

- Zwei unterschiedliche Metallurgien zu verschiedenen Perioden.

Die Abfallprodukte können simultan oder sukzessiv im Rahmen ein und derselben Technik entstanden sein:

- Ofenschlacken (intern abgekühlt) oder externe Fliesschlacken.

- Schlacken welche sich zu Beginn oder zum Schluss des Verhüttungsprozesses gebildet haben.

- Schlacken welche sich von der Ofenwand gebildet haben.

Üblicherweise ist die räumliche und stratigrafische Verteilung der Abfallprodukte, wie auch deren variablen Quantitäten, Voraussetzung um entsprechende Bildungsmodelle bestimmen zu können. Im vorliegenden Fall handelt es sich jedoch um oberflächliche Streufunde welche hierzu unzureichende Informationen liefern. Die chemisch-mineralogischen Zusammenhänge der gebildeten Gruppen, können aber Hinweise liefern: 
- die geschmolzenen Wandelemente und die nicht geschmolzenen Wandelemente müssen eine chemische und/oder elementare Übereinstimmung aufweisen.

- die verschiedenen Schlackentypen, welche der Verhüttung eines Eisenminerals entstammen, müssen Gemeinsamkeiten mit dem ausgehenden Eisenmineral aufweisen.

Der Vergleich der GDS und GPS ist daher für das Verständnis des Ausgangsmaterials von Bedeutung.

Die GDS bilden eine makroskopisch, mineralogisch und chemisch homogene Gruppe.

Die GPS lassen sich ebenfalls als Gruppe definieren, sind jedoch weniger homogen. Man beobachtet makroskopische Farbvariationen, Mischvarianten, die partielle Präsenz von gewissen Mineralen und eine relativ signifikante chemische Variation innerhalb dieser Gruppe.

Um diese Koexistenz zu erklären bieten sich zwei Hypothesen an:

1. Man könnte sich vorstellen, dass an diesem Ort erst ein Rennofen bestanden hat, welcher Schlacken des Typs GDS produziert hat. Später wurde der Ofen zu einem Hochofen umgebaut, welcher Schlacken des Typs GPS hervorbrachte. Man kann sich aber auch vorstellen, dass derselbe Ofen teils als Rennofen, teils als Hochofen genutzt wurde, je nach dem ob Schmiedeisen oder Gusseisen gefragt war. In beiden Fällen stammen die Rückstände vom selben Eisenmineral $\mathrm{ab}^{2}$ und müssten daher Gemeinsamkeiten aufweisen.

2. Die zweite Hypothese ist, dass die GDS von Rückständen der Eisenmineralverhüttung stammen und die GPS von der Verschlackung der Ofenwand stammen. In diesem Fall müssten die beiden Gruppen unterschiedliche Zusammensetzungen aufweisen.

\section{Zusammenfassend:}

\section{Hypothese:}

Die Schlackentypen GDS und GLS stammen von demselben Mineral, aber von unterschiedlichen Produktionsmethoden (Renn- resp. Hochofen).

\section{Hypothese:}

Die Schlackentypen GDS und GLS stammen von unterschiedlichen Materialien ab (von einem Eisenmineral, resp. von der Ofenwand). 
Zur Klärung, ob die Schlacken von der Verhüttung desselben Minerals abstammen, kann man die Verbindungen zwischen den chemischen Elementen untersuchen, welche sich nicht reduzieren lassen, d.h. jene Elemente, welche sich integral in der Schlacke ablagern, sich aber nicht mit dem Metall verbinden. Für diese Elemente müssten die Verbindungen identisch sein.

Dabei ist zu beachten, dass die metallurgische Industrie diverse Substanzen verwendet $^{3}$, welche zu Verunreinigungen führen kann (Kontanimation der Ofenwand oder über die Holzkohlenasche).

Man kann das Diagramm von Ellingham verwenden (Schema 1), um das Verhalten der chemischen Elemente während der Reduktion nachzuvollziehen. Es können dabei die chemischen Oxyde bestimmt werden, welche in der Eisenmetallurgie niemals reduziert werden können (weniger reduktiv als Silizium: $\mathrm{TiO}_{2}, \mathrm{CeO}_{2}, \mathrm{Al}_{2} \mathrm{O}^{3}$, $\mathrm{MgO}, \mathrm{CaO}, \mathrm{Zr}, \mathrm{Rb}, \mathrm{Y})$, jene welche etwas reduktiver als Eisenoxyd sind $\left(\mathrm{P}_{2} \mathrm{O}_{5}, \mathrm{Cr}_{2} \mathrm{O}_{3}\right.$, $\mathrm{MnO}, \mathrm{V}_{2} \mathrm{O}_{3}$ ) und jene, die weniger reduktiv sind als Eisenoxyd ( $\mathrm{CoO}, \mathrm{NiO}, \mathrm{PbO}$, $\left.\mathrm{CuO}, \mathrm{Ag}_{2} \mathrm{O}\right)$

\section{Die Hauptelemente}

\section{$\mathrm{Fe}_{2} \mathrm{O}_{3}$}

Eisen $\left(\mathrm{Fe}_{2} \mathrm{O}_{3}\right)$ kommt in der Natur reichlich vor.

Der gemessene Eisengehalt der GDS ist erhöht (55 bis $79 \%$ ), höher als in den meisten Schlacken welche aus Rennöfen stammen.

Der Eisengehalt in den GPS ist tief (4 bis $11 \%$ ) und ähnlich wie jener in den untersuchten Tonfragmenten (5 bis $9 \%$ ). In den Abfällen moderner Hochöfen findet man oft Anteile welche noch tiefer liegen (1 bis $3 \%$ ), in alten Hochöfen werden meist Werte im Bereich 5 bis $15 \%$ gemessen. In primitiven Hochöfen können diese Werte bis zu $25-30 \%$ ansteigen.

\section{$\mathrm{SiO}_{2}$ und $\mathrm{Al}_{2} \mathrm{O}_{3}$}

Das Verhältnis zwischen $\mathrm{SiO}_{2}$ und $\mathrm{Al}_{2} \mathrm{O}_{3}$ ist ein Indikator zum Verständnis der verwendeten metallurgischen Systeme. Es handelt sich um zwei nicht reduzierbare Elemente. Sie sind in den meisten natürlichen Felsgesteinen vorhanden, namentlich in den Graniten. Sie können durch einen künstlichen Zusatz (Sand, Tone, etc.) oder durch die Ofenwand kontanimiert werden. Es gibt zudem immer einen Beitrag an $\mathrm{SiO}_{2}$ welcher von der Holzkohlenasche stammt, dieser ist aber im Vergleich zum Beitrag welcher vom Mineral oder der Ofenwand abstammt unbedeutend.

3 meist ein kalkhaltiger Schmelzzusatz. 
Da es sich hier um die beiden häufigsten Elemente handelt (nach dem Eisen), die in den Schlacken vorkommen, sind sie für die Interpretation von grosser Bedeutung. Eine Interpretation welche im Widerspruch zu den Messungen von $\mathrm{SiO}_{2}$ und $\mathrm{Al}_{2} \mathrm{O}_{3}$ steht, ist problematisch. Dennoch genügen diese beiden Elemente nicht immer für eine ausreichende Interpretation.

In den GDS ist Silizium mässig erhöht (15 bis $25 \%$ ), wie auch Aluminium (5 bis 10 \%). Der Gehalt beider Elemente ist ähnlich. Das Verhältnis variiert zwischen 2.5 und $3.5 \%$. Diese Variation kann vom verwendeten Eisenmineral abhängen oder von einer Kontanimation der Ofenwand stammen.

Ohne Analyse des verhütteten Eisenminerals kann man die Variation des Minerals weder ausschliessen noch bestätigen. Generell ist das Ausmass dieser Variation nicht überraschend. Das Verhältnis 2.5- 3.5 \% ist üblich in granitischem Material im weitesten Sinne ${ }^{4}$. Das Resultat steht also nicht im Widerspruch zum dominanten Felsgestein der Region, welches das in Frage stehende Eisenmineral beinhalten könnte. Hingegen lässt sich stark aluminiertes Mineral ${ }^{5}$ ausschliessen wie auch ein Mineral das nur schwach Aluminiumanteile aufweist ${ }^{6}$.

Vergleicht man diese Werte mit den beiden gebrannten Tonproben (2.87 und 3.25 $\%)$, kann festgestellt werden, dass sich hier die Verhältnisse wiederholen. Diese Situation ist allerdings schwierig zu interpretieren. Eine Kontanimation durch die Ofenwand kann weder bestätigt noch ausgeschlossen werden.

Interessant ist, dass die GPS generell etwas höhere Werte, zwischen 3 und $3.8 \%$ aufweisen ${ }^{7}$. Dennoch liegen die Werte nur leicht erhöht und daher nicht wirklich verschieden von den vorgenannten.

Aufgrund dieser Beobachtung lässt sich nicht bestätigen, dass die GDS und GPS von zwei verschiedenen Ausgangsmaterialien (vor der Fusion) abstammen, aber es besteht eine gewisse Wahrscheinlichkeit.

Es besteht eine Anreicherung an Silizium um das dreifache ${ }^{8}$ und des Aluminiums um das $21 / 2$-fache. Diese leichte Differenz könnte eine Kontanimation durch die siliziumhaltige Ofenwand (s. weisses Tonfragment, H100) bedeuten, oder aber auch Folge der geringen Beprobung sein.

\footnotetext{
4 Die Granite selbst, jedoch auch derivate Felsgetseine wie Gneis, metamorpher Schiefer oder sandigem Sediment, schwach arkosisch, d.h. noch mit Feldspatanteilen.

5 Meist Lateriten, Verhältnis 1:1.

$6 \mathrm{Im}$ Verhältnis $>5$.

7 Auch im Verhältnis zu den üblichen granitischen Werten.

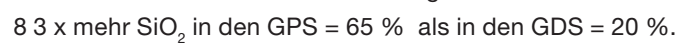


$\mathrm{TiO}_{2}$

Titan ist ein nicht reduzierbares Element. Es kommt in den meisten natürlichen Materialien vor, mit schwachem Gehalt ( 0.1 bis $1 \%$ ). Es sind seltene Eisenminerale (Ilmenit) in welchen Titan angereichert vorkommt, wie auch in gewissen (schweren) Sanden.

Der Titangehalt in den GDS variiert zwischen 0.1 und $0.3 \%$, in den GPS und den Tonen liegt der Anteil bei 0.6 und $0.7 \%$, also deutlich höher. In beiden Schlackengruppen ist die Verbindungen zwischen $\mathrm{SiO}_{2} \mathrm{zu} \mathrm{TiO}_{2}$ und $\mathrm{Al}_{2} \mathrm{O}_{3} \mathrm{zu} \mathrm{TiO}_{2}$ nicht signifikant verschieden (aber auch nicht identisch).

Diese Beobachtung erlaubt keine Entscheidung zwischen beiden Hypothesen. Der Faktor Anteil an $\mathrm{TiO}_{2}$-Gehalt ist etwa 3-fach, wie auch jener von $\mathrm{SiO}_{2}$.

\section{$\mathrm{MnO}$}

Das Mangan (MnO) ist ein Element, das bei Temperaturen eines Rennofens sich nicht reduzieren lässt, im Hochofen jedoch eine Reduktion erfahren kann. Es handelt sich um ein Element, das in den meisten Felsgesteinen vorkommt (0.1 bis $1 \%$ ). Es ist in gewissen Eisenmineralen angereichert (reduzierte, mit FeO angereicherte Minerale und Minerale aus Oberflächenverwitterung).

Der Gehalt an MnO bei den GDS beträgt 0.3 bis $0.9 \%$, während bei den GPS und Tonen nur 0.05 bis $0.1 \%$ nachgewiesen werden konnten.

Diese Beobachtung ist mehrdeutig und erlaubt keine Bestätigung oder Verwerfung der Arbeitshypothesen.

Der $\mathrm{MnO}$-Gehalt in den GDS ist ein Hinweis, dass das verhüttete Eisenmineral etwas $\mathrm{MnO}$ beinhaltete und dieses in die Schlacke gelangte, was für einen Rennofen üblich ist. Ein Gehalt im Eisenmineral von 0.5\% könnte einen Anteil von 0.3 bis $0.9 \%$ an die Schlacken abgeben. Ein solch hoher Anteil an $\mathrm{MnO}$ in einem Eisenmineral hat nichts Überraschendes.

Der MnO-Gehalt in den GPS ist wesentlich geringer. Man kann dies damit erklären, dass die GPS aus der Fusion der tonig-sandigen Ofenwand stammt ${ }^{9}$. Eine weitere Erklärung wäre, dass die GPS das Resultat einer Hochofenverhüttung sind, unter Verwendung eines Eisenminerals mit schwachem MnO-Gehalt. In diesem Fall würde $\mathrm{MnO}$ reduziert und das $\mathrm{Mn}$ geht in das Metall über. Der MnO-Anteil in der Schlacke wäre dann gering. In diesem Fall könnte man erwarten, dass der Verringerungs-faktor des $\mathrm{Mn}$ weniger bedeutend ist als jener des Metalls ${ }^{10}$. Die Variationen der Resultate sind mehrdeutig. Eine Schlussfolgerung ist daher nicht möglich.

9 Der MnO Anteil der GPS ist mit jenem der gebrannten Tone kompatibel.

10 das wesentlich reduktiver ist als das $\mathrm{Mn}$. 


\section{MgO}

Magnesium (MgO) ist ein Element das nicht reduzierbar ist. Es ist zu unterschiedlichen Anteilen in den Felsgesteinen vertreten. In basischen und ultrabasischen kann der Anteil bis zu $20 \%$ betragen. In den granitischen Felsgesteinen beträgt der Anteil um 1 bis $2 \%$. Das MgO kann in gewissen karbonatisierten Felsgesteinen vorkommen. In Eisenmineralen ist $\mathrm{MgO}$ selten reichlich vorhanden.

MgO kann im Holz vorkommen und findet sich daher in der Holzkohlenasche wieder. Damit dies so ist, muss das Substrat reich an $\mathrm{MgO}$ sein, auf welchem sich das Holz entwickelt hat. Im vorliegenden Fall, ist das Substrat arm an MgO.

Der Gehalt an MgO in den GDS und in den GPS ist immer derselbe, zwischen 1.0 und $2.5 \%$. Auch bei den Tonen wurden dieselben Anteile gemessen.

Bei der ersten Hypothese, bei welcher die GDS und GPS von demselben Eisenmineral stammen, müssten die Anteile bei den GPS um 2 bis 3 mal höher sein, als bei den $\mathrm{GDS}^{11}$. Die gemessenen Anteile an $\mathrm{MgO}$ widersprechen aber dieser $\mathrm{Hy}-$ pothese.

Die gemessenen Anteile in den Tonen und GPS sind vergleichbar. Die Hypothese, dass die GPS das Resultat der Ofenwandfusion sind, ist nicht widerlegt.

Es ist kein Beitrag an MgO durch die Holzkohlenasche auszumachen, weil MgO keine Korrelation aufweist mit den anderen Elementen, im Speziellen mit CaO. Man bemerkt lediglich, dass der Gehalt an MgO in den Tonen höher ist als in den GPS. Dies könnte einen Zusammenhang mit der Holzkohlenasche bedeuten, aber der klare Beweis steht aus.

\section{$\mathrm{CaO}$}

Das Kalzium ist ein nicht reduzierbares Element. Es ist im Felsgestein in unterschiedlichem Gehalt präsent. Im Kalkgestein, auf Kalzitbasis $\left(\mathrm{CaCO}_{3}\right)$, kann es das Hauptelement sein. In anderen Felsgesteinen kann es mit einem Anteil von 1 bis $5 \%$ vorkommen. $\mathrm{CaO}$ ist in Eisenmineralen selten vorhanden. Ausnahme bilden sedimentäre, marine, oolitische Minerale.

$\mathrm{CaO}$ ist das wichtigste minerale Element das man im Holz findet und damit auch in der Holzkohlenasche, wo es zu 50 bis $90 \%$ präsent ist. Der Anteil hängt von zahlreichen Faktoren ab, im Wesentlichen von der Art des Holzes und dem Substrat.

Der CaO-Gehalt der GDS ist sehr tief ( 0.5 bis $1 \%$ ) und kann vom Mineral stammen. Eine Kontanimation durch die Holzkohle hat nur sehr schwach oder nicht stattgefunden.

In der Fundregion können kalziumarme Eisenminerale erwartet werden.

Der CaO-Gehalt bei den GPS ist noch tiefer oder equivalent. Wie auch für das $\mathrm{MgO}$, widerspricht diese Beobachtung der Hypothese eines Hochofens.

11 Anreicherungsfaktor für $\mathrm{SiO}_{2}, \mathrm{Al}_{2} \mathrm{O}_{3}, \mathrm{TiO}_{2}$, etc. 
In den Tonen sind die Anteile sehr tief (<0.5\%). Die Differenz zu den GPS liegt womöglich an einer Kontanimation mit Holzkohlenasche.

Die CaO-Anteile in den GPS sind zu tief um auf die Verwendung von kalkhaltigen Verhüttungszusätzen zu schliessen.

\section{$\mathrm{Na}_{2} \mathrm{O}$}

Das Sodium $\left(\mathrm{Na}_{2} \mathrm{O}\right)$ verflüchtigt sich bei hohen Temperaturen und ist in der Metallurgie daher meist nicht mehr nachweisbar. Mit der XRF-Analyse ist es schwer messbar, die Anteile sind nicht signifikant.

Im vorliegenden Fall lohnt sich eine Interpretation der Anteile nicht.

\section{$\mathrm{K}_{2} \mathrm{O}$}

Das Kaliumcarbonat $\left(\mathrm{K}_{2} \mathrm{O}\right)$ ist ein nicht reduzierbares Element und in allen Felsgesteinen vorhanden mit einem Anteil von ca. 0.5 bis $3 \%$. Selten kann es in Eisenmineralen nachgewiesen werden.

Wie auch das $\mathrm{CaO}$, zieht $\mathrm{K}_{2} \mathrm{O}$ in das Holz bis zu einem Gehalt von 10 bis $50 \%$. Mit Blick auf die Fundregion und dem granitischen Substratum, kann ein hoher Anteil an $\mathrm{K}_{2} \mathrm{O}$ im Holz erwartet werden ${ }^{12}$.

Der Gehalt an $\mathrm{K}_{2} \mathrm{O}$ bei den GDS beträgt 0.75 bis $2.25 \%$. Bei den GPS sind sie signifikant höher (zwischen 3 und $4 \%$ ). Die Werte der Tone bewegen sich zwischen den Gehaltsanteilen beider Gruppen.

Das Verhältnis $\mathrm{CaO}$ zu K $\mathrm{K}_{2} \mathrm{O}$ beträgt $0.5 \%$ bei den GDS und $0.2 \%$ bei den GPS. Bei der Hypothese, in welcher davon ausgegangen wird, dass GDS und GPS vom selben Eisenmineral abstammen, könnte man auch davon ausgehen, dass dieselbe Holzkohle verwendet wurde. Die beobachteten Resultate jedoch weisen nicht klar in diese Richtung. Allerdings kann man die Proportionen an Holzkohleasche, welche in die Schlacken eingedrungen ist, nicht bestimmen. Weil die Aschenproportionen zwischen Renn- und Hochofen bestimmt variieren, ist es also nicht möglich die Entwicklung des Gehaltes und des Verhältnisses $\mathrm{CaO} z u \mathrm{~K}_{2} \mathrm{O}$ vorzusehen.

Man kann also die $\mathrm{K}_{2} \mathrm{O}$-Resultate nicht dazu verwenden, um die erste Hypothese auszuschliessen. Hingegen macht die zweite Hypothese, bei welcher die GDS aus der Verhüttung stammen und die GPS aus geschmolzener Ofenwand entstanden sind, in diesem Fall Sinn.

Weil die Minerale normalerweise arm an $\mathrm{CaO}$ und $\mathrm{K}_{2} \mathrm{O}$ sind, kann man bei den GDS davon ausgehen, dass diese beiden Elemente von der Holzkohle aufgenommen wurden. In diesem Fall kann man das Verhältnis $\mathrm{CaO}$ zu $\mathrm{K}_{2} \mathrm{O}$ berechnen, was einem Faktor von 0.5 entspricht und als Beitrag der Holzkohle verstanden werden kann.

$\mathrm{K}_{2} \mathrm{O}$ überwiegt den Anteil des $\mathrm{CaO}$. Wenn man diesen Aspekt berücksichtig, erklärt 
sich die Differenz der $\mathrm{CaO}$ und $\mathrm{K}_{2} \mathrm{O}$-Anteile der Tone gegenüber der GPS. Bei den Tonen konnte ein Anteil an $\mathrm{CaO}$ von 0.4 und $2.7 \%$ an $\mathrm{K}_{2} \mathrm{O}$ nachgewiesen werden. Die GPS betragen einen Gehalt an $\mathrm{CaO}$ von $0.7 \%$ und an $\mathrm{K}_{2} \mathrm{O}$ von $3.4 \%$. Wenn man $0.3 \% \mathrm{CaO}$ den Tonen beimischt, könnte man GPS herstellen. In diesem Fall müssen $0.6 \% \mathrm{~K}_{2} \mathrm{O}$ beigefügt werden um den Anteil der Holzkohle auszugleichen ${ }^{13}$. Dieser Beitrag von 0.6 \% $\mathrm{K}_{2} \mathrm{O}$ der Holzkohle zu den $2.7 \% \mathrm{~K}_{2} \mathrm{O}$ in den Tonen gäbe $3.3 \%$, was sehr nahe bei $3.4 \%$ liegt, dem durchschnittlichen $\mathrm{K}_{2} \mathrm{O}$-Anteil bei den GPS. Die Resultate sind also kohärent.

\section{$\mathbf{P}_{2} \mathbf{O}_{5}$}

Phosphor ist ein Element, das sich knapp über dem Schmelzpunkt des Eisens reduzieren lässt. Im Rennofen erzielt man eine partielle Reduktion des Eisens, man wird somit auch eine partielle Reduktion des Phosphors erreichen. Phosphor wird sich also zwischen Metall und Schlacke verteilen. Im Hochofen erzielt man eine komplette Reduktion des Eisens, so auch des Phosphors, das sich ins Metall absetzt und nicht in die Schlacke.

Phosphor ist in niedrigen Quantitäten in allen Felsgesteinen vorhanden ( 0.5 bis $1 \%$ ). Phosphor kommt selten konzentriert vor. Die harten Partien eines lebenden Organismus (Zähne, Knochen) nutzen Phosphore (Apatit). Sedimente, welche aus einem hohen Anteil an Skeletten bestehen, sind mit Phosphor angereichert. Gewisse Eisenminerale bilden sich in Milieus, welche mit Phosphor angereichert sind. Dies ist vor allem bei marinen, oolitischen Mineralen der Fall. Man findet auch stark phosphorhaltige Minerale in periglacialen Milieus (Moore). Auch gibt es magmatische Minerale mit signifikanten Phosphoranteilen.

Das Holz hat wenig Phosphoranteil, der Gehalt beträgt lediglich 1 bis $5 \%$ und ist daher zu gering für eine Kontanimation und zu schwach um in der Schlackenanalyse nachgewiesen zu werden. Hingegen verwenden gewisse Kulturen stark phosphorhaltiges Heizmaterial, wie Knochen oder Yakmist.

Die GDS haben einen $\mathrm{P}_{2} \mathrm{O}_{5}$-Anteil von 0.7 bis $2 \%$. Dieser Gehalt ist normal für Rennofenschlacken und stammt von einem Mineral das einen normalen Phosphoranteil aufweist.

Es gibt daher keinen Grund anzunehmen, dass hier ein spezielleres Heizmaterial verwendet wurde.

Die GPS zeigen einen sehr schwachen $\mathrm{P}_{2} \mathrm{O}_{5}$-Anteil mit 0.1 bis $0.3 \%$. Diese Beobachtung wäre mit der Hypothese, welche den Hochofen postuliert, kompatibel. Sozusagen der gesamte Phosphoranteil ist reduziert und hat in das Metall gewechselt.

In den Tonen wurden $\mathrm{P}_{2} \mathrm{O}_{5}$-Anteile gemessen, welche jenen der GPS entsprechen. Man kann also die GPS auch als geschmolzene Tone betrachten, was wiederum der zweiten Hypothese nahe kommt.

$13 \mathrm{CaO}$ zu K $\mathrm{K}_{2} \mathrm{O}=0.5$ oder $\mathrm{K}_{2} \mathrm{O}$ zu CaO $=2$. 


\section{Die Spurenelemente}

Folgende Elemente wurden nicht nachgewiesen, weil sie zu schwach vorhanden sind, oder sie waren in den Proben nicht präsent: Sc, As, Se, Br, In, Ge, Sn, Sb, Te, Sm, Pt, Th und U.

Nebst den nachfolgend besprochenen Spurenelementen wurden auch Rb, Ag, Cu, $\mathrm{Zn}, \mathrm{Cd}, \mathrm{Ba}, \mathrm{Cr}, \mathrm{Ga}, \mathrm{In}, \mathrm{Y}, \mathrm{Bi}, \mathrm{Ce}, \mathrm{Hf}, \mathrm{V}, \mathrm{Nb}, \mathrm{S}, \mathrm{Mo}, \mathrm{Cl}, \mathrm{Ge}, \mathrm{Sn}, \mathrm{Sb}, \mathrm{Te}, \mathrm{Pt}, \mathrm{Au}$, Th und $\cup$ gemessen. Aufgrund zu geringer Anteile dieser Elemente, konzentrieren wir uns hier auf jene Spurenelemente deren Anteile eine gewisse Signifikanz aufweisen.

\section{Cs}

Das Cäsium ist ein nicht reduktives Element.

Es konnte sozusagen kein Cs Gehalt in den SGD nachgewiesen werden. In den GPS wurde jedoch ein Anteil zwischen 50 und 150 ppm gemessen. Bei den Tonen liegt der Anteil um 50 ppm.

Diese schwachen Anteile sind mit dem Programm UNIQUANT nicht mit grosser Präzision gemessen worden. Dennoch scheinen die Ergebnisse die Hypothese zu widerlegen, welche davon ausgeht, dass GDS und GPS vom selben Mineral stammen. Die schwachen Anteile an Cs weisen auf ein beinahe Cs-freies Mineral hin. Die höheren Anteile bei den GPS können sich aber nicht mit einem Cs-freien Mineral erklären lassen, und es ist schwierig einen anderen Zusammenhang zu finden, welcher zu diesem Resultat geführt hat.

Obwohl es sich um mittelmässige Resultate handelt, besteht eine Kohärenz mit der Hypothese die davon ausgeht, dass die GPS das Resultat geschmolzener Ofenwand sind.

$\mathrm{Sr}$

Das Strontium (Sr) ist ein nicht reduktives Element. Das Element ist oft assoziiert mit Kalzium, namentlich im Kalzit.

Die GDS haben einen Anteil von unter 100 ppm, die GPS liegen mit 150 ppm über diesen Werten, und die Tone wiederum weisen einen Wert von 100 ppm aus ${ }^{14}$. Bei der Hypothese, welche für GDS und GPS dasselbe Ausgangsmineral vorschlägt, würde man eine signifikantere Anreicherung von GDS und GPS erwarten. Die Resultate scheinen daher diese Hypothese zu widerlegen.

14 Diese schwachen Anteile sind mit dem Programm UNIQUANT nicht mit grosser Präzision gemessen worden. Dagegen ist Sr ein Element das sich mit der XRF-Analyse gut messen lässt. 


\section{Co}

Kobalt ist reduktiver als Eisen und ein Spurenelement welches mit gewissen Eisenmineralen assoziiert ist.

Die Anteile mit einigen Hundert ppm sind signifikant messbar. Die Messgenauigkeit ist bestimmt nicht sehr präzis, es lassen sich dennoch vertrauenswürdige Resultate erkennen.

Das Tungstenmetall aus welchem die Steinmühle gefertigt ist und mit welcher die Proben gemahlen wurden, beinhaltet Kobalt und kann daher Rückstände bilden. Im gemessenen Fall jedoch ist der Co-Anteil signifikant und kann nicht vom Mahlprozess alleine stammen.

Die GDS weisen einen Co-Anteil auf von 300 bis 400 ppm. Dies ist viel für ein Element das eine komplette Reduktion hätte erfahren sollen. Wir haben hierfür keine plausible Erklärung.

Man stellt fest, dass die Probe H115a, welche makroskopisch viel Metall enthält, auch am meisten Kobalt beinhaltet. Man kann davon ausgehen, dass der CoAnteil der Übertragung von Kobalt ins Metall entspricht.

In den GPS ist der Gehalt an Co geringer (100 bis 200 ppm). Bei der Rennofenhypothese dürften keinerlei Co-Rückstände erwartet werden.

Bei den Tonen sind die Anteile variabel (70 und 250 ppm), was der Spannweite der GPS entspricht.

Insofern wäre die Hypothese, dass die GPS geschmolzene Ofenwände sind, mit diesen Werten kohärent.

\section{$\mathrm{Ni}$}

Nickel (Ni) ist reduktiver als Eisen und ein Spurenelement, welches mit gewissen Eisenmineralen assoziiert ist. Die Anteile sind sehr schwach und nicht wirklich signifikant.

Man stellt fest, dass 6 von 7 GDS-Proben kein Ni beinhalten, wie man dies von einem vollkommen reduktiven Element erwarten kann.

Im Fall der Probe H115a, besteht ein sichtbar hoher Anteil an Eisen. Die dort gemessenen 250 ppm Ni könnten ihre Präsenz während der metallischen Phase erklären.

Die GPS und die Tone beinhalten einige 10 ppm Ni. Bei einer Hochofenverhüttung wären diese Anteile zu hoch, zudem weisen die GDS eine komplette Reduktion des Ni auf. Die gemessenen Werte des Ni verwerfen daher die Hochofenhypothese und stützen jene, welche die Verschmelzung der Ofenwand vorschlägt.

\section{$\mathrm{Pb}$}

Blei $(\mathrm{Pb})$ ist ein leicht reduzierbares Element, dessen Verhalten jedoch noch wenig beschrieben ist. Die Fusion erfolgt bei niedrigen Temperaturen $\left(800^{\circ} \mathrm{C}\right)$. Man stellt fest, dass das $\mathrm{Pb}$ nicht in die Schlacken übergeht, aber es ist nicht bekannt ob es 
ins Metall eindringt. Unter der Bedingung, dass genügend Material vorhanden ist, könnte es eventuell separat eine flüssige Phase bilden. In der Natur ist Blei mit $\mathrm{Cu}$, Zn und $\mathrm{S}$ vergesellschaftet und kommt in Eisenmineralen nicht vor ${ }^{15}$.

Die GDS beinhalten kein Blei, was entweder bleifreies Mineral indiziert oder bedeuten kann, dass das $\mathrm{Pb}$ ins Metall, oder in eine andere Phase metallischer Verflüssigung übergegangen ist.

Die Anteile in den GPS sind sehr gering. Die Tone weisen ähnliche Werte auf.

Diese Werte sind mit der Hochofenhypothese nicht kompatibel ${ }^{16}$. Dagegen sind die Bleianteile in den GPS durchaus mit der Hypothese der geschmolzenen Ofenschlacken vereinbar.

\section{La}

Lanthan (La) ist ein nicht reduzierbares Element. Es ist in schwachen Mengen im Felsgestein vorhanden. Es gibt keine Affinität zu gewissen Eisenmineralen.Die gemessenen Anteile sind sehr tief und nicht präzis messbar.

Der La-Anteil in den GDS ist abnorm hoch (bis 80 ppm). Die Anteile in den GPS und den Tonen sind beinahe null oder sehr tief (<20 ppm).

Diese Angaben sind nicht präzis, aber die Abweichungen scheinen dennoch bedeutsam und argumentativ. Die Werte sind mit der Hochofenhypothese nicht vereinbar, denn sonst müsste La in die GPS konzentriert übergegangen sein, während sie im vorliegenden Fall dort nur sehr schwach nachgewiesen werden konnten. Dieses Argument verweist klar in die zweite Hypothese.

$\mathrm{Zr}$

Zirkonium (Zr) ist völlig reduzierbar. Es ist im Felsgestein schwach präsent, mit einer Affinität für siliziumhaltiges Gestein, dort meist als Zirkon $\left(\mathrm{ZrSiO}^{4}\right)$ vorhanden, und kann konzentriert auch in gewissen Sanden auftreten. Es gibt keine besondere Affinität zu Eisenmineralen.

Die gemessenen Werte sind zwar sehr tief, dennoch kann eine akzeptable Messpräzision erwartet werden. Es ist ein Element das sich gut über die XRF-Analyse messen lässt.

In den GDS wurden Werte von 50 bis 150 ppm gemessen. Die Werte korrelieren positiv mit $\mathrm{SiO}_{2}, \mathrm{TiO}_{2}$ und $\mathrm{Al}_{2} \mathrm{O}_{3}$, negativ hingegen mit $\mathrm{Fe}_{2} \mathrm{O}_{3}$. Ein Hinweis, dass die drei Elemente von derselben Quelle stammen, vermutlich vom Mineralgang. Die Korrelation zu $\mathrm{MgO}$ und $\mathrm{CaO}$ ist schwach ausgeprägt, zwei Elemente die eher mit der Holzkohle in Verbindung stehen.

Diese Werte bestätigen die Hypothese, welche die GDS als Rennofenschlacken bezeichnet. Zudem kann ein Zr-armes Eisenmineral vermutet werden.

Bei den GPS und den Tonen sind die Zr-Anteile höher (zwischen 300 und 500

15 Mit Ausnahme der Schwefelminerale.

16 Das gesamte Blei müsste von der Schlacke getrennt sein. 
ppm). Es besteht eine ziemlich gute Korrelation mit Silizium, jedoch keine mit $\mathrm{Al}_{2} \mathrm{O}_{3}$ noch mit $\mathrm{TiO}_{2}$. Man bemerkt aber auch eine negative Korrelation mit MgO. Dieses Verhalten ist mit einem Hochofen nicht vereinbar.

Die Anreicherung beträgt das 3- bis 5-fache, was allerdings für einen Hochofen nicht abwegig ist.

Der Vergleich der Werte in den Tonen und den GPS stützt wiederum die Hypothese der Ofenwandverschlackung.

\section{Ta}

Das Verhalten von Tantal ist noch ungenügend beschrieben. Ta ist vermutlich schwer reduzierbar, wie zum Beispiel auch $\mathrm{Nb}$, und ist nicht speziell mit Eisenmineral liiert.

Die Anteile in den GDS sind variabel und teilweise auch erhöht (0 bis 150 ppm). Bei den GPS und Tonen betragen die Werte immer Null.

Es gibt keine Grundlagen dies zu interpretieren, aber im Prinzip scheint dies seltsam, dass dieses Element im Mineral präsent sein kann, aber in den GDS nicht vorkommt, als wären diese unter Hochofenbedingungen reduziert worden, und $\mathrm{Ta}$ demnach auch nicht in den GPS vorhanden ist. Gleichzeitig scheint es logisch zu sein, dass das Element nicht in den GPS erscheint, wenn diese das Resultat der Ofenwandverschlackung sind, denn die Tone weisen ebenfalls kein Ta nach.

\section{w}

Das Verhalten von Tungsten/Wolfram (W) ist noch ungenügend beschrieben. Es ist nahe am Fusionspunkt des Eisens reduzierbar und steht $\mathrm{Nb}$ und Ta sehr nahe. Es gibt keine spezielle Verbindung mit den Eisenmineralen.

Das Material der Mahlmaschine, mit welcher die Proben zermahlen wurden, beinhaltet hohe Anteile an Wolfram. Eine Kontanimation von einigen 10 ppm ist daher normal.

Die gemessenen Werte sind jedoch sehr hoch (100 bis 1'000 ppm). Dies kann a priori nicht alleine auf das Mahlwerkzeug zurückgeführt werden. Die Kontanimation müsste die Gesamtheit der Proben betroffen haben, welche zudem nicht grosse Härteunterschiede aufweisen. Weil es mehr Quarz in den GPS hat, dürften dies die härtesten Stücke gewesen sein, aber die poröse Textur hat vermutlich das Zermahlen erleichtert.

Bei den GDS sind die Anteile an W eher tief, mit 100 - 300 ppm. Bei den GPS wurden höhere Werte von 300 - 700 ppm gemessen. Die beiden Tone ergeben Werte von 300 - 1100 ppm.

Diese Werte sprechen sich eher gegen die Hochofenhypothese aus, denn in einem Hochofen müsste Wolfram bei den GDS reduziert sein und die Werte bei den GPS deutlich tiefer liegen. Ein Hinweis welcher sich daher für die zweite Hypothese, jene der Ofenwandverschlackung, ausspricht. 


\section{Konklusion der chemischen Analyse}

Alle interpretierbaren Werte der GPS zeigen, dass diese mit der zweiten Hypothese kompatibel sind. Dies bedeutet, dass die GPS durch Verschlackung der Ofenwände entstanden sind. Die wenigen Abweichungen sind als Kontanimation der Holzkohlenasche erklärbar.

Für mehre Elemente stehen die gemessenen Werte im Widerspruch zu den Vorgaben der ersten Hypothese, bei welcher davon ausgegangen wird, dass am selben Ort mit zwei unterschiedlichen Verhüttungstechniken immer dasselbe Mineral reduziert worden wäre. Den Rennofenkonditionen würden GDS, den Hochofenkonditionen GPS resultieren.

Die Elemente welche dies belegen sind $\mathrm{MgO}, \mathrm{CaO}, \mathrm{Cs}, \mathrm{Sr}, \mathrm{Ni}, \mathrm{Pb}$, La und W.

Für $\mathrm{CaO}$ und $\mathrm{Sr}$ kann eine Variation des Heizmaterials die Ursache sein und also mit der Kontanimation durch Holzkohlenasche zusammen hängen.

Bei MgO könnte man sich ebenfalls eine Kontanimation über die Asche vorstellen, aber die hohen Werte sind für diese Annahme dennoch zu hoch.

A priori lässt sich die Beitragsvariation aus Holzkohlenasche nicht ausschliessen, aber sie ist weniger plausibel (und auch weniger einfach zu erklären) als die Stabilität der Asche, denn es gibt keinen Grund, weshalb das Heizmaterial während der Produktion hätte geändert werden sollen.

Für Ni und La sind die Resultate nachvollziehbar. Für Cs, Pb und W sind Einwände berechtigt.

Man kann natürlich auch die Hypothese aufstellen, dass ein unbekanntes Eisenmineral mit einer unbekannten Verhüttungsmethode reduziert wurde. Diese Erklärung ist jedoch weniger einfach nachzuvollziehen und daher auch weniger wahrscheinlich.

Es gibt kein Argument, dass Schmelzzusätze eingesetzt wurden. Die chemischen Resultate sind durch die mineralogischen Untersuchungen bestätigt worden.

\section{Bemerkungen zum Heizmaterial}

Die Annahme, dass Steinkohle als Heizmaterial verwendet wurde, ist nicht vertretbar. Der regionale, geologische Kontext ist für Steinkohlevorkommen eher ungünstig und die Verkehrswege wären hierfür ungeeignet, ein Import undenkbar. Es gibt sehr viel Wald in der Fundregion. Denkbar, dass diese in früherer Zeit noch dichter bewaldet war. Der Bedarf an Heizmaterial konnte somit problemlos vor Ort gedeckt werden. 
Es bieten sich nicht viele Argumente an, ob Holzkohle oder ungeköhlertes Holz für die Verhüttung verwendet wurde. Es gibt makroskopische Abdrucke in vielen Schlacken und die Entdeckung kleinerer Holzkohlestücke im Inneren der Schlakkenprobe H107a.

Die grossen Abdrucke, von einigen $10 \mathrm{~cm}$ Länge, sind ein klarer Beweis für die Nutzung von Holz (ohne jedoch die ausschliessliche Nutzung von Holz zu postulieren). Die zahlreichen kleinen Abdrucke sprechen für die Nutzung von Holzkohle (aber auch hier ist keine Ausschliesslichkeit vertretbar). Aber überzeugend ist das nicht, denn wir haben zuwenig Proben um hier eine abschliessende Beurteilung abgeben zu können und schliesslich endet jedes Holz, das in den Ofen gebracht wurde, früher oder später als kleines Holzkohlenstückchen.

\section{Bemerkungen zu den Produktions- mengen}

Die Berechnung der Produktionsmengen hängt sehr von der Ausgiebigkeit des Eisenminerals ab. Leider liegen keine Proben dieses Minerals vor. Zudem handelt es sich hier um die Beprobung einer Oberflächenprospektion, weshalb wir auch keine Kenntnis haben vom Schlackenvolumen, welcher beim Fundort noch im Boden liegt.

\section{Bemerkungen zum verwendeten Eisenmineral}

Wie bereits erwähnt, liegen keine Proben vor, mit welchem das verhüttete Eisenmineral hätte bestimmt werden könnte. Über die chemischen Untersuchungen der Schlacken lässt sich das gesuchte Mineral allenfalls eingrenzen.

Man kann davon ausgehen, dass es sich um ein besonders reiches Mineral gehandelt hat. Die untersuchten Schlacken weisen ca. $64 \% \mathrm{Fe}_{2} \mathrm{O}_{3}$ auf. Man müsste daher ein Mineral mit mehr als $65 \% \mathrm{Fe}_{2} \mathrm{O}_{3}$ erwarten. Bei weniger als $70 \%$ Anteil, wäre die Produktion äusserst gering.

Das verwendete Mineral ist silizium-aluminös und beinhaltet etwas $\mathrm{MgO}$, aber sehr 
wenig $\mathrm{CaO}$ und $\mathrm{K}_{2} \mathrm{O}$. Es hat etwas $\mathrm{MnO}(0.5 \%)$, dieser Anteil ist aber zu gering, um auf ein Eisenmineral zu schliessen.

Es beinhaltet etwas $\mathrm{P}_{2} \mathrm{O}_{5}$ (mehr als $0.1 \%$, vermutlich weniger als $1 \%$ ), was bereits auf ein phosphorhaltiges Mineral weist.

Die typischen Spurenelemente ( $\mathrm{Cr}, \mathrm{V}, \mathrm{Ni}, \mathrm{Cu}$, etc.) sind immer nur mit schwachen Anteilen vertreten (oder nicht vertreten, wie im Fall von $\mathrm{Ni}$ ). Es handelt sich somit nicht um eine typisiertes Erz nach Zitzmann.

Aufgrund des zu hohen Verhältnisses von $\mathrm{SiO}_{2} \mathrm{zu} \mathrm{Al}_{2} \mathrm{O}_{3}$, können die kontinentalen, aus Oberflächenverwitterung stammenden Minerale (Laterit) ausgeschlossen werden. Ebenso können die marinen, oolitischen Minerale ausgeschlossen werden, aufgrund der zu tiefen Anteilen an $\mathrm{CaO}$ und $\mathrm{P}_{2} \mathrm{O}_{5}$.

Man kann schwefelhaltige Adern, mangels Schwefelnachweis, und deren oberflächlichen Ausbildungen, wie Eisenhüte, ebenso ausschliessen, weil diese zuwenig Zeit hatten sich im Himalaja auszubilden.

Man kann Bändererz ausschliessen, weil der Anteil an $\mathrm{Al}_{2} \mathrm{O}_{3}$ dafür zu hoch wäre. Magnetit Anhäufungen des Typs Skarn können nicht ausgeschlossen werden, aber es fehlen die beweisführenden Indizien hierfür, wie $\mathrm{Sn}$, Ba, etc.

Der Abbau von Hämatitadern in granitischem Umfeld ist sehr wahrscheinlich. Der Gehalt eines solchen Minerals hängt hauptsächlich von der mechanischen Trennung des Hämatits vom umgebenden Felsgestein ab. Man kann einen hohen Gehalt erwarten, wenn gering angereicherte Felsblöcke verwendet werden. 


\section{Bemerkungen zur Verhüttungstemperatur}

Mit Blick auf das Phasendiagramm (Schema 1) dürften bei den GDS die Temperaturen 1 ' $250{ }^{\circ} \mathrm{C}$ erreicht haben. Die Anteile an $\mathrm{CaO}$ und $\mathrm{K}^{2} \mathrm{O}$ sind tief und haben auf die Fusion keinen signifikanten Einfluss gehabt.

Bei den GPS ist Vorsicht geboten, denn ein hoher Anteil an $\mathrm{SiO}_{2}$ ist nicht geschmolzen. Hier müssten unter dem Elektronenmikroskop weitere Untersuchungen folgen, um verlässliche Rückschlüsse auf die Verhüttungstemperaturen ziehen zu können.

Schema 1:

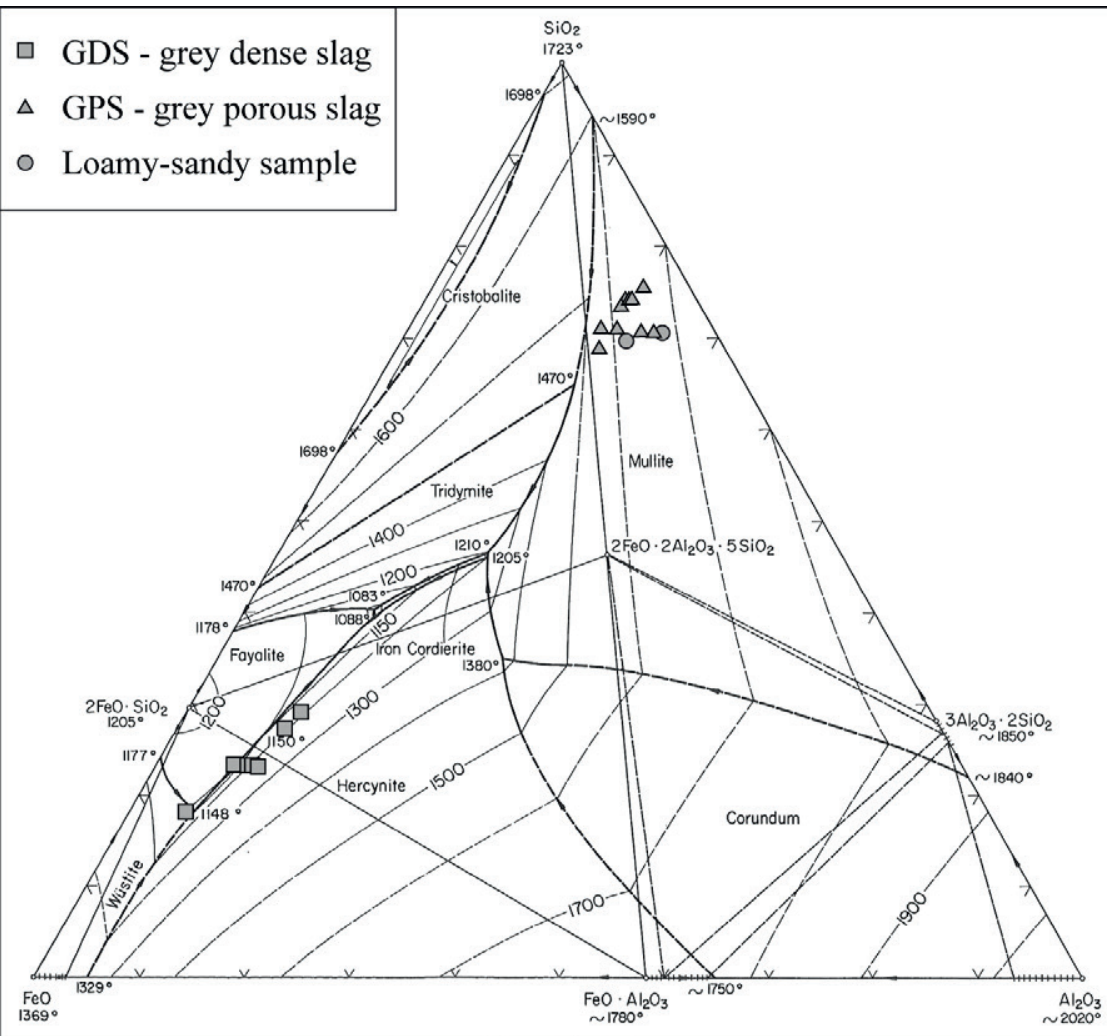



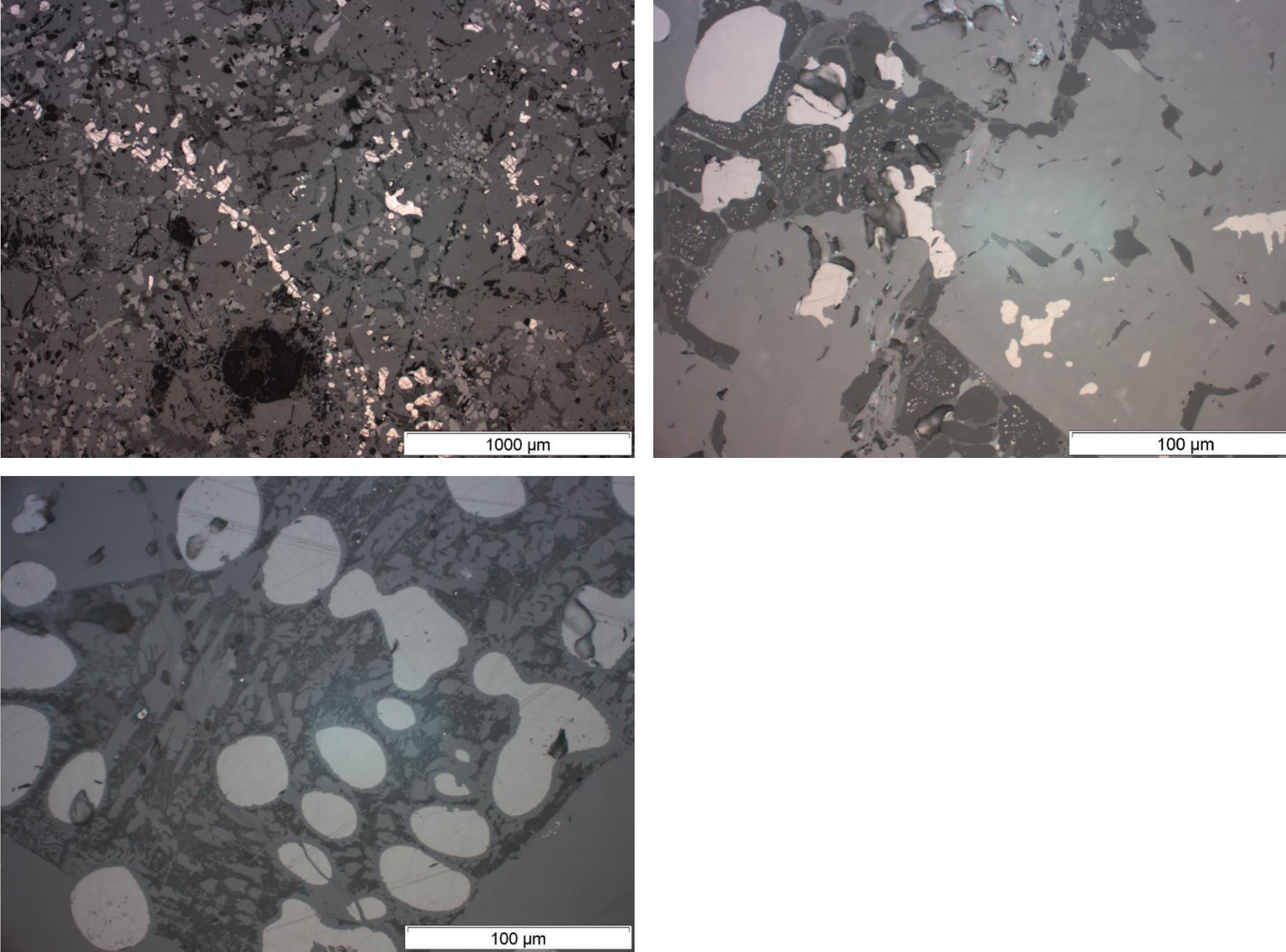

Abb. 13:

Probe H45. Dend-

ritische Wüstite und vereinzelt Metallstreifen.

Abb. 14:

\section{Untersuchung der optischen} Mikroskopie

Probe H45. Spinelle und Leucite.

Es wurden zwei Dünnschliffe der GDS und 1 Dünnschliff für die GPS angefertigt.

Abb. 15:

Probe $\mathrm{H} 45$.

\section{GDS/Probe $\mathrm{H} 45 \mathrm{a}$}

Stäbe aus Fayalit

zweiter Generation.

Homogener Schliff mit grossen, gedrungenen, eckigen fayalitischen Kristallen, mit teilweise massiertem Aufkommen. Generell befindet sich wenig Glas in den Zwischenräumen. Viele dendritische Wüstite und vereinzelt Metallstreifen (Abb. 13). Einige helle Spinelle (Abb. 14).

Man findet zwei verschiedene Texturen in den Zwischenräumen der Fayalite: Dunkle Leucitkörner, welche um die Dendrite der Wüstite gewachsen sind und als kleine weisse Punkte sichtbar sind (Abb. 14); feine Stäbe aus zweiter Fayalitgeneration, deren Metall von der Zersetzung der Wüstitkörner stammt (Abb. 15). 

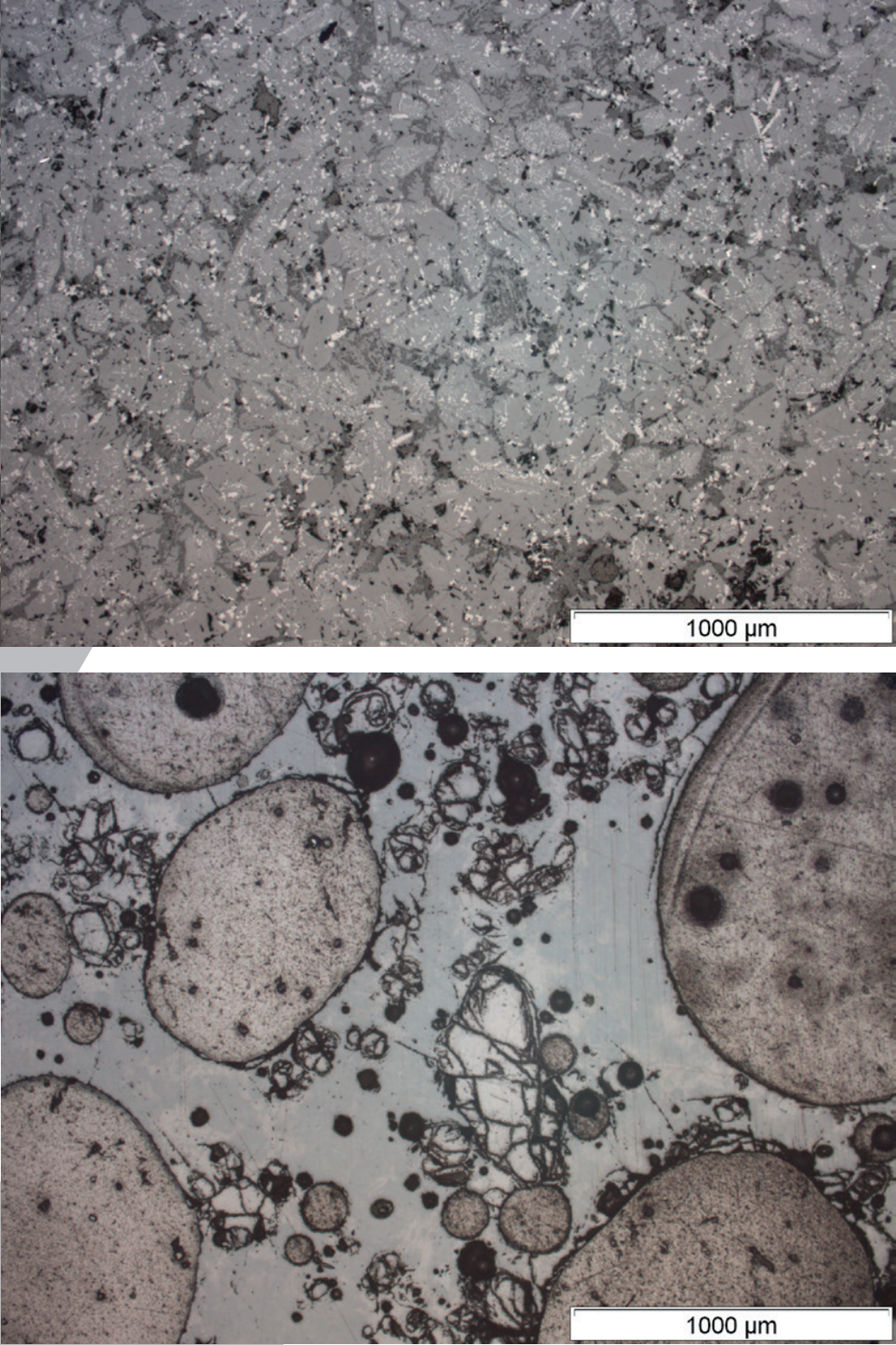

Abb. 18:

Probe H108. Spinelle mit Leucite vergesellschaftet.

Abb. 19:

Probe H104f. Quartz.

Abb. 20:

Probe H104f. Kristallisiertes Mullit.

\section{Abb. 21:}

Probe H104f. Kristallisiertes Mullit, polarisiertes Licht.
In den hellen und dunkleren Zonen ist der Schliff homogen. Man beobachtet eine grosse Porosität in einer glasigen Matrix, welche sekundär eingeschlossenes, nicht geschmolzenes Quarz beinhaltet (Abb. 19). Näher betrachtet beinhalten die verglasten Zonen feine, zu Stäben kristallisiertes Mullit (Abb. 20), welches in polarisiertem Licht eine faserige Struktur bildet (Abb. 21).

Keine Eisenoxyde, kein Fayalit sichtbar. Die Farbdifferenz, von hell bis dunkler, entstand aufgrund unterschiedlicher Dauer der Materialabkühlung. 
52 


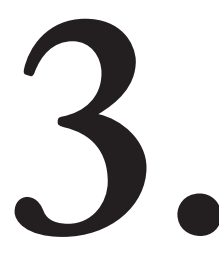

\section{Die Resultate der ${ }^{14} \mathrm{C}$-Analyse}

Irka Hajdas, Dominique Oppler

Beim Schnitt der Schlackenprobe H107a fanden sich gut erhaltene, in der Schlakke eingeschlossene Holzkohlenstücke. Ebenso hafteten an der Aussenseite der Probe Na7 kleine, feine Holzkohlenstücke. Die Holzkohlen aus diesen beiden Proben wurden im ${ }^{14} \mathrm{C}$-Labor der ETH-Zürich datiert.

Resultatetabelle der ${ }^{14} \mathrm{C}$-Datierung

\begin{tabular}{l|l|l|l|l} 
Lab Nummer & $\begin{array}{l}\text { Proben } \\
\text { Code }\end{array}$ & Material & $\begin{array}{l}{ }^{14} \mathbf{C} \text { Alter } \pm 1 \sigma \\
(\mathbf{B P})\end{array}$ & $\begin{array}{l}\boldsymbol{\delta}^{13} \mathbf{C} \pm \mathbf{1 \sigma} \\
\left(\%^{\circ}\right)\end{array}$ \\
\hline ETH-46924 & $\mathrm{H} 107 \mathrm{a}$ & Holzkohle & $823 \pm 27 \mathrm{BP}$ & $-24.3 \pm 1.1$ \\
ETH-46923 & $\mathrm{Na} 7$ & Holzkohle & $332 \pm 26 \mathrm{BP}$ & $-27.0 \pm 1.1$
\end{tabular}

Nachfolgende Angaben sind mit OxCal v3.10 kalibriert und in Kalenderjahre umgerechnet worden. 


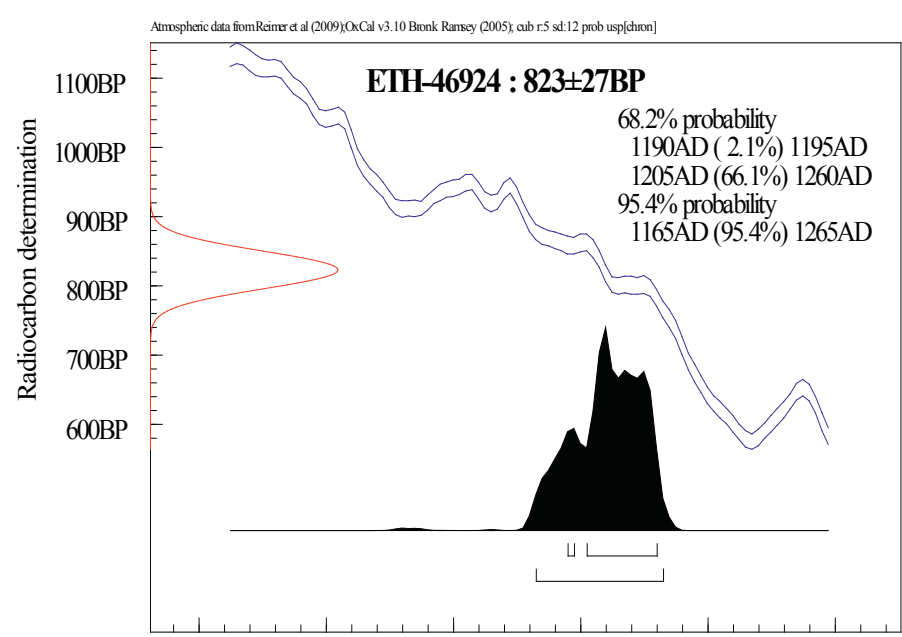

900CalAD 1000CalAD 1100CalAD 1200CalAD 1300CalAD 1400CalAD Calibrated date

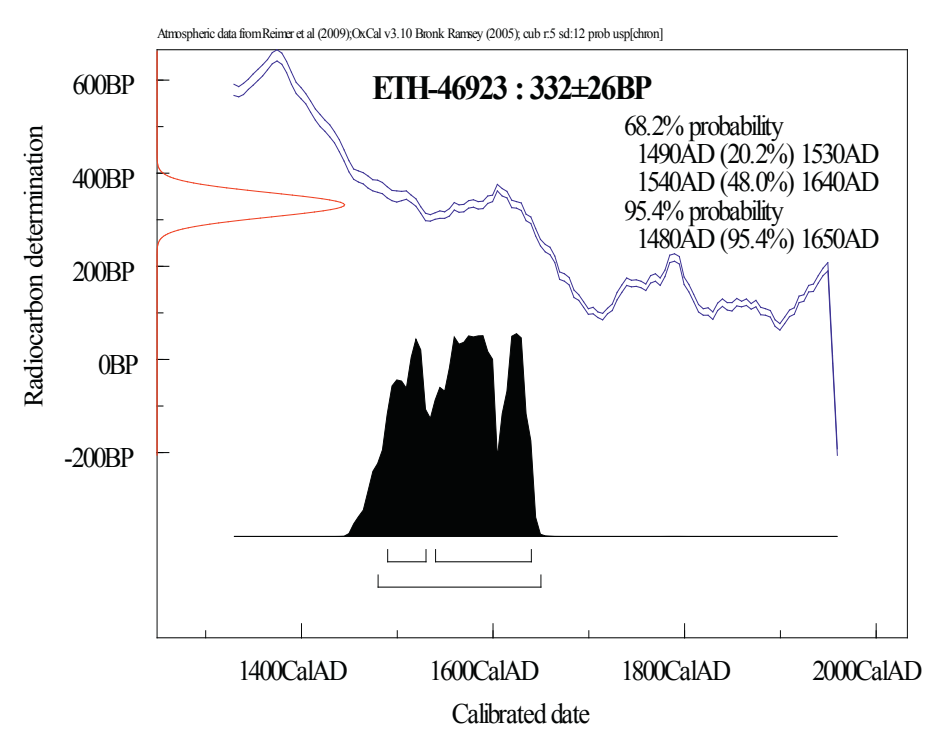

Abb. 22:

${ }^{14} \mathrm{C}$-Diagramme der

Holzkohlenproben

$\mathrm{H} 107 \mathrm{a}$ (links) und $\mathrm{Na}$ / (rechts).)

Im 1 Sigma-Bereich (68.2 \% Vertrauensbereich) stehen folgende Zeitspannen zur

Diskussion:

$$
\begin{aligned}
& 1490 \text { - } 1530 \text { n.Chr. (20.2 \%) } \\
& 1540 \text { - } 1640 \text { n. Chr. ( } 48 \%)
\end{aligned}
$$

Im 2 Sigma-Bereich (95.4 \% Vertrauensbereich) steht die Zeitspanne 1480 - 1650

n. Chr. zur Diskussion.

\section{Datierung der Probe aus H107a}

Im 1 Sigma-Bereich (68.2 \% Vertrauensbereich) stehen folgende Zeitspannen zur Diskussion:

$$
\begin{aligned}
& 1190 \text { - } 1195 \text { n. Chr. (2.1\%) } \\
& 1205 \text { - } 1260 \text { n. Chr. (66.1\%) }
\end{aligned}
$$

Im 2 Sigma-Bereich (95.4 \% Vertrauensbereich) 1165-1265 n. Chr. zur Diskussion. 


\section{Konklusion der ${ }^{14} \mathrm{C}$-Datierungen}

Die Datierung der Probe Na7 übereinstimmt mit weiteren Datierungen organischer Materialien der Grabung auf dem Drapham-Dzong die zuvor beim Centre for Isotope Research ( $\mathrm{ClO}$ ) in Groningen untersucht wurden. Wir können daher davon ausgehen, dass die Schlacke vor der Zerstörung des Drapham-Dzongs gebildet wurde. Die kalottenartige Schlacke könnte einer Schmiedeesse entstammen. Dass auf einer Burg geschmiedet wurde ist nicht weiter erstaunlich.

Auf den ersten Blick überrascht die frühe Datierung der Holzkohle aus der Schlakke H107a. Es ist durchaus denkbar, dass bereits einige Zeit vor Errichtung des Drapham-Dzong beim Weiler Nag Lhakhang Eisen verhüttet wurde. Wenn man der Legende um Pema Lingpa Glauben schenkt, bereits zwischen 1450 und 1521. Diese Schlackenprobe datiert aber noch um einiges älter.

Erst seit den 70er-Jahren wird in Bhutan, und dies namentlich auch in der Provinz Bumthang, der Wald professionell genutzt und forstwirtschaftlich gepflegt (Urech Zora, 2009, S. 12ff.). Mit Ausnahme einiger mächtiger immergrünen Eichen, ist der Baumbestand seither verjüngt worden. Im 16./17. Jahrhundert jedoch dürfte der Busch noch recht unberührt gewesen sein und die metallurgische Industrie nutzte womöglich auch älteres bis sehr altes Holz. Dies mag eine Erklärung sein, weshalb die ${ }^{14} \mathrm{C}$-Datierung bei der Schlackenprobe H107a um ca. 370 Jahre ältere Werte misst. Diese beiden Hypothesen schliessen sich gegenseitig nicht aus.

Aufgrund der nicht repräsentativen Beprobung, können diese Datierungen nur informativen Charakter haben und erlauben keinerlei Interpretation. 


\section{Schlussfolgerung}

Wir wissen nicht, ob die Eisenverhüttung bei Nag Lhakhang mit dem DraphamDzong und dessen Reichtum in Verbindung stand. Um hierzu eine schlüssige Antwort zu erhalten, müsste der Verhüttungsplatz zumindest mit ein paar Sondierschnitten untersucht und systematischer beprobt werden können. Die Erlaubnis hierzu hatten wir nicht und auch nicht die Zeit und Ressourcen, welche dazu notwendig gewesen wären.

Bei der Schlacke Na7, welche auf dem Drapham-Dzong gefunden wurde, könnte es sich um eine Kalotte oder Schmiedeschlacke handeln, aber sicher ist dies nicht. Die Schlacke ist sehr stark mit Sand und sonstigem Erdmaterial vermengt und weist keine Struktur auf, welche das Stück als Kalotte eindeutig ausweisen würde. Ein Zusammenhang zwischen den Schlacken des Drapham-Dzongs und jenen des Verhüttungsplatzes, konnte anhand der Probe Na7 nicht eindeutig hergestellt werden. Es liesse sich allenfalls anhand einer vergleichenden Analyse der Metallobjekte, welche im Laufe der Grabungskampagne gefunden wurden, einen Zusammenhang nachweisen. Die dazu erforderlichen Methoden sind invasiv und aufwendig und machen erst Sinn, wenn der Verhüttungsplatz repräsentativ untersucht worden ist. Wenn wir der ${ }^{14} \mathrm{C}$-Datierung folgen, und der Hypothese Glauben schenken, dass sehr altes Holz geköhlert wurde, dann ergibt sich möglicherweise eine Verbindung zum Dzong. Aber das gilt es erst noch zu beweisen.

Das Mineral, welches in Nag Lhakhang verhüttet worden ist, kennen wir nicht. Die chemischen und mineralogischen Untersuchungen konnten jedoch eine gewisse Eingrenzung aufzeigen, und sprechen sich für ein hämatithaltiges Gestein aus.

Bei den vorliegenden Proben kann mit Sicherheit davon ausgegangen werden, 
dass ausschliesslich Eisen verhüttet worden ist und keine Schwer- oder Edelmetalle.

Ob das Eisenmineral aus der Region stammt, ist schwer zu beurteilen, geologisch spricht aber einiges dafür. Capt. R. Boileau Pemberton, ein britischer Gesandter, erwähnt in seinem Bericht von 1839 zudem einen Eisenmineralabbau bei Tassgong (Tongsa): [...] Speere und Pfeilspitzen werden in Tongsa hergestellt, von Eisen das in den Bergen gewonnen wird, am nördlichen Fuss des Schlosses, und kupferne Kessel werden geformt von Metall, von dem es heisst, dass es in den Bergen am Fusse von Tassangsee [?] gewonnen wird, der Ort wird in ganz Bhutan für sein überlegenes Handwerk gefeiert. Die Entfernung von Tongsa nach Nag Lhakhang oder dem Drapham-Dzong beträgt ca. 30 km-Luftlinie (heute ca. 3 Std. Autofahrt). Von derselben Quelle (Boileau Pemberton R., 1839 (Rpr. 2005), S. 79). erfährt man aus der Import und Exportstatistik der Karawanen von damals, dass von Bhutan Silber importiert und Kupfer exportiert wurden, Eisen fehlt aber sowohl auf der einen wie auch auf der anderen Statistik.

Die chemisch-mineralogischen Analysen haben ergeben, dass höchstwahrscheinlich mit einem Rennofen verhüttet wurde, bei Temperaturen von ca. 1'250 $\mathrm{C}$. Vermutlich wurde Holzkohle verwendet, aber den sicheren Beweis hierfür haben wir nicht.

Über die Bauart des Rennofens lässt sich nur spekulieren. Eine Grabung würde hierüber Aufschluss geben. Wir wissen nur, dass Natursteine verwendet wurden und im Inneren des Ofens die Wände vermutlich mit Lehm ausgekleidet waren, so wie dies auch andernorts üblich war.

Wie die Belüftung funktionierte wissen wir nicht. Eine Probe $(\mathrm{H} 100)$ könnte aufgrund der Materialbeschaffenheit und einer leicht konvexen Verformung, auf eine Düse hinweisen, aber das ist reine Spekulation.

Dass mit Holz gefeuert wurde ist erwiesen. Ob dieses geköhlert wurde, dafür sprechen einige Beobachtungen, aber erwiesen ist es nicht. Auch besteht keine Möglichkeit einen Nachweis zu erbringen, dass die Kohle tatsächlich an Ort und Stelle hergestellt wurde, beispielsweise auf der erwähnten Plattform.

Die Produktionsmengen zu bestimmen ohne Kenntnis zu haben vom verwendeten Ausgangsmineral, ist nicht möglich. Die Schlackenhalde weist teils besonders mächtige, schwere, stark oxydierte, eisenhaltige Schlackenblöcke auf. Leider waren diese für den Transport und den Rückflug nach Europa zu schwer und hätten die Frachtkosten zusätzlich in die Höhe getrieben. Aber auch bei den zur Verfügung stehenden Proben konnte festgestellt werden, dass die Verhüttung nur sehr ungenügend gelungen ist. Das kann mit zu niedrigen Temperaturen, problematischer Bauart des Ofens, oder aber mit einem besonders reichhaltigen Mineral zusammenhängen - trotz schwacher Verhüttung deckte die Ausbeute dennoch den Bedarf. 


\section{Bibliographie}

- Aris Michael (1979), Bhutan, the early history of a Himalayan kingdom, Warminster, England.

- $\quad$ Aris Michael (1994), The raven crown, The origins of Buddhist monarchy in Bhutan, London.

- Aris Michael; Ardussi John A. (2009), Sources for the history of Bhutan, New Delhi.

- Beer Robert (2003), The Handbook of Tibetan Buddhist Symbols, 1st ed., Boston, Mass.

- Benoit, Paul (Hg.) (1995), Paléométallurgie du fer \& cultures, Actes du symposium international du Comité pour la Sidérurgie Ancienne de l'Union Internationale des Sciences Préhistoriques et Protohistoriques ; Belfort-Sévenans, Institut Polytechnique de Sévenans, 1-2-3 novembre 1990, Belfort, Paris.

- Bernier Ronald M. (1997), Himalayan Architecture, Madison, London.

- Boileau Pemberton R. (1839), Report on Bootan, Repr. 2005, Calcutta.

- $\quad$ Craddock Paul T. (1995), Early metal mining and production, Edinburgh.

- Gansser, Augusto (1983), Geology of the Bhutan Himalaya, Basel.

- Gerner Manfred (2007), Chakzampa Thangtong Gyalpo - Architect, Philosopher and Iron Chain Bridge Builder, Thimphu.

- $\quad$ Gyatso J. (1981), A literary transmission of the traditions of Thang-stong rGyalpo, A study of visionary buddhism in Tibet, Berkeley.

- International Symposium on 'The Archaeometallurgy of Iron (1987), Results Achieved 1967-1987, held at Liblice, Czechoslovakia.

- $\quad$ LaRocca Donald J. (2006), Warriors of the Himalayas, Rediscovering the arms 
and armor of Tibet ; [Exhibition "Warriors of the Himalayas: Rediscovering the Arms and Armor of Tibet" on view at The Metropolitan Museum of Art, New York, April 5 - July 2, 2006], New Haven, Conn.

- $\quad$ Linduff Katheryn M. (2004), Metallurgy in ancient eastern Eurasia from the Urals to the Yellow River, Lewiston, NY.

- Lo Boe E., Statuary metals in Tibet and the Himalayas, in Oddy W.A. and Zwalf W. (Hg.), Aspects of Tibetan Metallwork, British Museum Occasional Paper, London: British Museum15, Bd. 15. 33-67.

- Mayhew Bradley (2011), Bhutan, 4. Aufl., London.

- Mei Jianjun (2000), Copper and bronze metallurgy in late prehistoric Xinjiang, Its cultural context and relationship with neighbouring regions, Oxford.

- Mei Jianjun; Rehren Thilo (2009), Metallurgy and civilisation, Eurasia and beyond : proceedings of the 6th International Conference on the Beginnings of the Use of Metals and Alloys (BUMA VI), London.

- $\quad$ Nils Anfinset (2011), An Ethnoarchaeological Study from Nepal., Social and Technological Aspects of Mining, Smelting and Casting of Copper.

- $\quad$ Oddy W.A. and Zwalf W. (Hg.) (1981), Aspects of Tibetan Metallwork, British Museum Occasional Paper Bände, London.

- O'Rourke, The Stratigrafy of Himalayan Iron Ores, in Rodgers John (Hg.), Americam Journal of Science, Etsablished in 1818 by Benjamin Silliman, New Haven, Conn. [u.a.]. Bd. 260. 294-302.

- Phelchey Toenkhyim (2006), The Folk Heritage Museum, Traditions of Bhutan, Thimphu.

- Prakash B. (1987), Methods of Iron Making in Early india, in, Inetrnational Symposium on 'The Archaeometallurgy of Iron'. Results Achieved 1967-1987, held at Liblice, Czechoslovakia.

- $\quad$ Ray Priyadaranjan (1956), History of chemistry in ancient and medieval India incorporating the History of Hindu chemistry by Acharva Prafulla Chandra Ray, Calcutta.

- $\quad$ Reedy Chandra L. (1997), Himalayan bronzes, Technology, style, and choices, Newark.

- $\quad$ Rubin Han Ko T. (1990), Ancient Iron and Steel Technology in China, in Benoit, Paul (Hg.), Paléométallurgie du fer \& cultures, Actes du symposium international du Comité pour la Sidérurgie Ancienne de I'Union Internationale des Sciences Préhistoriques et Protohistoriques ; Belfort-Sévenans, Institut Polytechnique de Sévenans, 1-2-3 novembre 1990, Belfort, Paris: Vulcain; Association pour l'Édition et la Diffusion des Études Historiques111-117.

- Thinley Namgyel; Karma Tenzin (2009), A Photo Guide to Flowers of Bhutan, Thimphu. 
- Varahmihira (1956), History of Chemistry in Ancient and Mideval India, Calcutta.

- Zitzmann (1977), The iron deposits of Europe, Vol. I. S. 13 - 35.

\section{Internet:}

- $\quad$ iron chain bridge (2006), Online verfügbar unter http://www.wolf-kahlen.net/ tibet-archive-berlin/seite15.htm, zuletzt aktualisiert am 04.02.2006 zuletzt geprüft am 09.07.2011.

- ScienceDirect - International Journal of Coal Geology : Gondwana coals of Bhutan Himalaja - Occurrence, properties and petrographic characteristics, Online verfügbar unter http://www.sciencedirect.com/science/article/ pii/0166516288900183 zuletzt geprüft am 10.07.2011.

- The stratigraphy of Himalayan iron ores -- O'Rourke 260 (4): 294 -- American Journal of Science (1962), Unter Mitarbeit von O‘Rourke und J. E, Online verfügbar unter http://ajsonline.org/cgi/content/abstract/260/4/294, zuletzt aktualisiert am 01.04.1962 zuletzt geprüft am 10.07.2011. 


\section{Anhang}

\section{Angewandte chemisch-physikalische Methoden zur Analyse des Schlacken- materials aus Bhutan}

Die angewandten Methoden ergänzen sich komplementär und dienen der Charakterisierung des untersuchten Materials. Sie haben zum Ziel die Zusammensetzung derchemischen Elemente (XRF) und die mineralogische Zusammensetzung (XRD) zu bestimmen, wie auch deren strukturelle Wirkung auf das Material zu verstehen (Mikroskopie). Die ${ }^{14} \mathrm{C}$-Datierungen wurden an der ETH Zürich ausgeführt, alle übrigen Untersuchungen erfolgten im Labor der Geoscience der Universität Fribourg.

\section{XRF (Röntgenfluereszenzanalyse)}

Nach dem die Schlacken gereinigt und eine Auswahl zur vertieften Analyse getroffen wurde, sind die Proben zu Pulver zermahlen worden (Mühle mit Elementen aus Tungstenstahl). Das Puder wurde zu Pastillen gepresst und im Spektrometer bestrahlt. Das Fluereszenzspektrum wurde durch das Softwareprogramm UNI- 
QUANT interpretiert welches halbwegs quantitative Resultate liefert zur Komposition der chemischen Elemente (Haupt- und Spurenelemente). Es werden nur Elemente gemessen, welche schwerer sind als $\mathrm{Na}$. Die Richtwerte betragen $0.1 \%$ für Elemente mit hohem Anteil (> 1\%). Für Spurenelemente liegt die Nachweisschwelle bei $0.01 \%$ (100 ppm). Die Resultate bedürfen daher einer vorsichtigen Interpretation. Die Verluste durch Erhitzung sind separat gemessen worden.

\section{Labor:}

Labor des Geologischen Departementes der Universität Freiburg (CH).

\section{Apparat:}

Spektrometer Philips PW2400, Tube PW2592/35 3kW Rhodium Anode

\section{Analyseprogramm:}

UNIQUANT von Thermoscientific - http://www.uniquant.com/

Die Analaysen wurden ausgeführt von:

Vincent Serneels, Raphaelle Soulignac und Dominique Oppler

\section{Bibliographie:}

- Jenkins, R. (1999) X-Ray Fluorescence Spectrometry, Wiley, 232p.

- $\quad$ Beckoff, B., Kanngiesser, B., Langhoff, N., Wedell, R., Wolff, H. (2006) Handbook of Practical X-Ray Fluorescence Analysis, Springer, 863p.

\section{XRD (Röntgendiffraktionsanalyse)}

Die Proben wurden zu Pulver verarbeitet (gleiches verfahren wie bei XRF). Eine Prise wurde auf dem Probenhalter fixiert und im Diffraktometer bestrahlt.

Jedes Mineral besitzt ein spezifisches Diffraktionsspektrum. Das globale Spektrum ist die Gesamtheit aller bestehnder Minerale. Die kristallinen Hauptbestandteile können nachgewiesen werden. Geringe Bestandteile (<5\%) sind schwer nachzuweisen.

Amorphe oder glasige Materie gibt keine Spektren ab, führt jedoch zu Erhöhung des Grundrauschens.

\section{Labor:}

Labor des Geologischen Departementes der Universität Freiburg $(\mathrm{CH})$. 
Apparat:

Diffraktometer Philips PW 1710, Tube Cu (40 kV et 40 Ma), CuK $\beta$ mit GraphitMonochromator.

Analysekonditionen:

Abtasten des Spektrums zwischen $5^{\circ}$ und $80^{\circ} 2$, (step $0.02^{\circ}, 1 \mathrm{sec} / \mathrm{step}$ ).

Die Analaysen wurden ausgeführt von:

Raphaëlle Soulignac und Dominique Oppler

\section{Bibliographie:}

- Als-Nielsen, J., McMorrow D. (2001) Elements of Modern X-ray physics, Wiley \& Sons.

- Warren B.E. (1990) X-ray Diffraction, General Publishing Company.

\section{Optische Mikroskopie}

Mit einer Diamantsäge wurden Stücke von $2 \times 3 \mathrm{~cm}$ den Schlacken entnommen. Danach wurden diese Proben auf eine geringe Dicke nachgeschnitten. Der Dünnschliff wurde daraufhin durch Einschleifen und anschliessendem Polieren erzielt. Die optische Mikroskopie mit übertragenem und reflektiertem Licht erlaubt bei hoher Vergrösserung die Struktur des Materials (Grösse und Form der Körnung, Korndichte, etc.). Es ist möglich Substanzen an ihrem optischen Aspekt und Eigenheiten (Farben) zu erkennen.

Labor:

Labor des Geologischen Departementes der Universität Freiburg $(\mathrm{CH})$.

\section{Mikroskope:}

Mikroskop Olympus GX51 mit gedrehter Platine (reflektiertes Licht) und Mikroskope Leica DMRXP (übertragenes Licht).

\section{Die Analysen wurden durchgeführt von:}

Raphaëlle Soulignac und Dominique Oppler

\section{Bibliographie :}

- Tröger, W.E. (1979) Optical Determination of Rock Forming Minerals, Schweizerbart. 


\section{Angewandte ${ }^{14} \mathrm{C}$-Datierungsmethode}

\section{Probenbearbeitung:}

Die Proben wurden zur Reinigung mit der Acid Alkali Acid Methode (AAA) behandelt (Hajdas 2008, Hajdas et al. 2004). Mit dieser Behandlung wurde die Holzkohle in ein schwach saures $\mathrm{Bad}(0.5 \mathrm{M} \mathrm{HCl})$ bei $60^{\circ} \mathrm{C}$ für $12 \mathrm{Std}$. eingetaucht um die Proben von Karbonaten zu reinigen. Danach wurden die Proben mehrfach mit distilliertem Wasser in einer schwachen Base $(0.1 \mathrm{M} \mathrm{NaOH})$ bei $60^{\circ} \mathrm{C}$ während 12 Std. durchtränkt. Dieser Prozess reinigt von humosen Säuren welche die Holzkohle allenfalls kontanimiert haben. Danach wurden die Proben erneut mit distilliertem Wasser mehrfach gereinigt um einen neutralen $\mathrm{pH}$-Wert nach der $\mathrm{NaOH}$ Behandlung zu erreichen. Eine letzte Säure-Reinigung entfernte atmosphärischen Kohlenstoff, welcher sich mit der Holzkohle währen den ersten Rinigungsprozessen gebunden hat. Danach wurden die getrockneten Proben (1 mg von C) graphitiert (Vogel et al.1984).

Es wurde das automatisierte AGE Graphitisationssystem (Wacker et al. 2010) angewandt, mit integrierter Elementanalyse zur Erzeugung von gereinigtem $\mathrm{CO} 2$ ( 1 mg C) der Holzkohleproben (2.5-3.5 mg).

Das CO2 wurde isoliert und in einen Reaktor transferiert welcher $3 \mathrm{mg}$ beschleunigtes Eisen beinhaltete (Alfa Aesor-325 Wicklung, mit Sauerstoff vorgeheizt und mit Hydrogen reduziert bei $\left.500^{\circ} \mathrm{C}\right)$. Hydrogen wurde hinzugefügt ( $\left.\mathrm{H} 2 / \mathrm{CO} 2 \approx 2.3\right)$ und die Mischung während $2 \mathrm{Std}$ aufgeheizt auf $580^{\circ} \mathrm{C}$. $\mathrm{H} 2 \mathrm{O}$ mitsamt Graphit wurden mit Hilfe eines Peltier Elementes eingefrohren.

Das Graphitpulver wurde danach in einen Probenhalter gepresst und mit dem Beschleunigermassenspektrometer MICADAS (AMS) der ETH Zürich (Synal et al. 2007) analysiert, parallel mit einem Set leerer und standardisierter Proben. Die untergrund (Blank) Proben bestanden aus ${ }^{14} \mathrm{C}$-freier Kohle und wurden dazu eingesetzt die Kontaminierung während des Aufbereitungsprozesses zu überwachen. Das konventionelle ${ }^{14} \mathrm{C}$-Alter wurde berechnet und beschrieben nach Stuiver and Polach (1977). Diese Datierungen beinhalten Korrekturen für Fraktionierungen welche auf semi-simultanous Messungen von ${ }^{13} \mathrm{C}$ des Graphites basieren. Die 1-Sigma-Fehlerquote beinhaltet Messunsicherheiten wie auch die Streuung der Standard- und Blankproben.

Das ${ }^{13} \mathrm{C}$-korrigiertes Radiokarbonalter basiert auf der 14C-Konzentration welche in den Proben gemessen wurde. $\left({ }^{13} \mathrm{C}\right.$ ist ein Wert, der bei Graphit gemessen wurde und der zusätzliche Fraktionierungen beinhalten kann.)

${ }^{14} \mathrm{C}$-Datierung mit OxCal v 3.10 kalibriert nach Bronk Ramsey, C. (2005) und INTCAL09 data set (Reimer et al., 2009). Alle Angaben verstehen sich BP vor heute (before present/before1950 AD). 
Labor:

14C-Labor für lonenstrahlphysik der ETH-Zürich.

\section{Apparate:}

Graphitisation mit AGE System (Wacker et al 2010).

14C-Analysen mit MICADAS (Synal et al. 2007).

\section{Die Analysen wurden durchgeführt von:}

Irka Hajdas, Dominique Oppler

\section{Bibliographie:}

- Hajdas, I. (2008), The Radiocarbon dating method and its applications in Quaternary studies, Quat. Sci. Jour.I - Eiszeitalter und Gegenwart 57, 2-24.

- Hajdas, I. et al. (2004), A report on sample preparation at the ETH/PSI AMS facility in Zurich, Nuclear Instruments \& Methods in Physics Research Section B-Beam Interactions with Materials and Atoms 223-24, 267-271.

- $\quad$ Reimer, P.J. et al. (2009), IntCal09 and Marine09 radiocarbon age calibration curves, 0-50,000 years cal BP, Radiocarb 51, 1111-1150.

- $\quad$ Stuiver, M. \& Polach, H.A. (1977)., Discussion: reporting of 14C data, Radiocarb, 19, 355-363.

- $\quad$ Synal, H. A., Stocker, M., Suter, M. (2007), MICADAS: A new compact radiocarbon AMS system, Nuc. Inst. \& Meth. in Phy. Res. Sec. B-Beam Inter. with Mat. \& Atoms 259, 7-13.

- Vogel, J. S., Southon, J. R., Nelson, D. E. \& Brown, T. A. (1984), Performance of Catalytically Condensed Carbon for Use in Accelerator Mass-Spectrometry, Nuc. Inst. \& Meth. in Phy. Res. Sec. B-Beam Inter. with Mat. \& Atoms 233, 289-293.

- Wacker, L., Nemec, M. \& Bourquin, J. (2010), A revolutionary graphitisation system: Fully automated, compact and simple, Nuc. Inst. \& Meth. in Phy. Res. Sec. B-Beam Inter. with Mat. \& Atoms 268, 931-934.

- Bronk Ramsey, C. (2005), OxCal v. 3.10. http://c14.arch.ox.ac.uk/embed php?File1/4oxcal.html 
This publication is also available open-access on

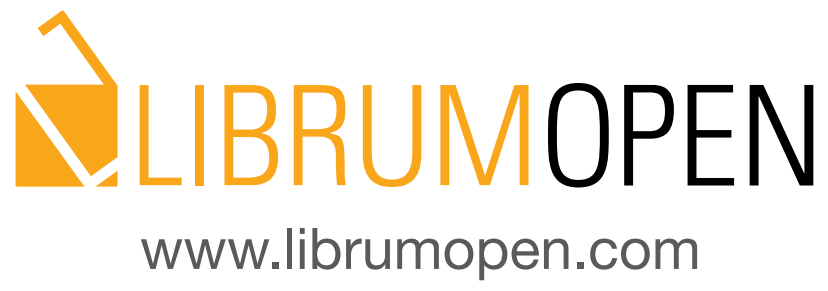

or as printed version at

\section{SliBRUMSTORE www.librumstore.com}

